Portland State University

PDXScholar

1983

\title{
The Japanese/American interface : a crosscultural study on the approach to discourse
}

Hitomi Tamura

Portland State University

Follow this and additional works at: https://pdxscholar.library.pdx.edu/open_access_etds

Part of the Discourse and Text Linguistics Commons, Japanese Studies Commons, and the Speech and Rhetorical Studies Commons

Let us know how access to this document benefits you.

\section{Recommended Citation}

Tamura, Hitomi, "The Japanese/American interface : a crosscultural study on the approach to discourse" (1983). Dissertations and Theses. Paper 3350.

https://doi.org/10.15760/etd.5231

This Thesis is brought to you for free and open access. It has been accepted for inclusion in Dissertations and Theses by an authorized administrator of PDXScholar. Please contact us if we can make this document more accessible: pdxscholar@pdx.edu. 
AN ABSTRACT OF THE THESIS OF Hitomi Tamura for the Master of Arts in Speech Communication presented May 16, 1983.

Title: The Japanese/American Interface: A Crosscultural Study on the Approach to Discourse.

APPROVED BY MEMBERS OF THE THESIS COMMIT TEE:

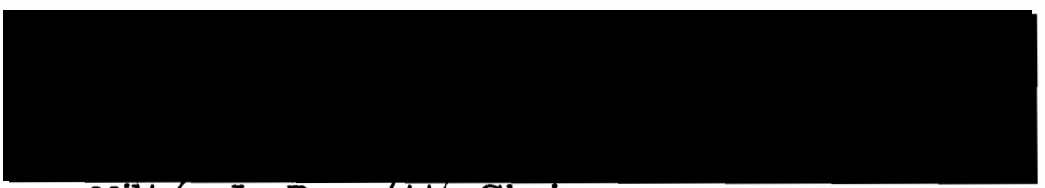

Milten J. Bennett, Chairman

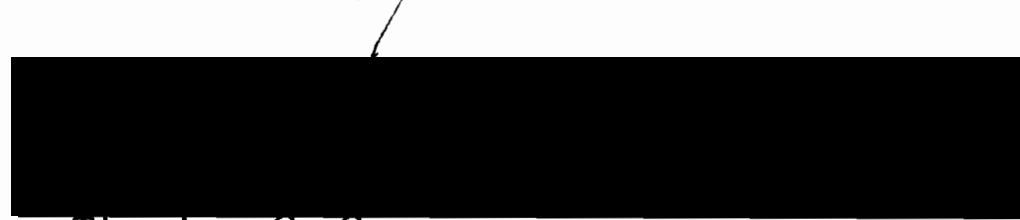

Theodore G. Grove

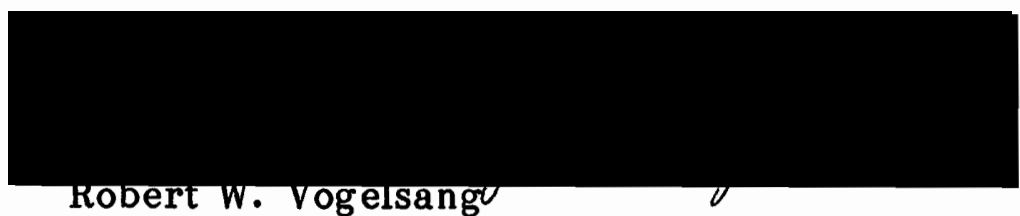

kovert w. vogelsang

This study attempted to explore one aspect of the communicative styles of Japanese and Americans: their approach to discourse. In a literature review, four distinctive characteristics were surveyed: linear/nonlinear presentation, inductive/deductive reasoning, explicit/implicit communication, and analytical/emotional statements. The American style of argument was characterized by:

1) a linear presentation as evidenced by its preference for a sequential paragraph development, its reliance on logic, and 
its direct introduction of the subject.

2) either inductive or deductive reasoning.

3) explicit communication as shown by its emphasis on the use of concrete language, definite qualifiers, clearly stated conclusions and a wider perspective.

4) analytical and objective statements.

The Japanese style of argument is characterized by:

1) its nonsequential presentation, noncontiguous paragraphs, its apparent disregard of logic and indirect introduction of the subject.

2) neither inductive nor deductive reasoning.

3) implicit communication as shown by its emphasis on the use of ambiguous language, the frequent use of conditional qualifiers, implied conclusions and a narrower perspective.

4) emotional and subjective statements.

The literature review corroborated the author's hypotheses that Japanese express themselves in an ambiguous manner, whereas Americans express themselves in a more clearly defined manner. The differences of style of arguments were tested in a statistical set ting using content analysis of current newspapers.

The results indicated that significant differences were observed in the degree of linearity in organization, the use of reasoning, relative directness of introduction of the subject, width of perspective, use of the definite qualifiers and objectivity of statements. No significant difference, however, was observed in repeated number of conclusions, concreteness and analyticity of statements and degree of explicitness of conclusion. Crosscultural homogeneity was observed among editorials 
from the two groups.

Thus, there was substantial evidence to assume that American style of discourse is predominantly clear, whereas Japanese style of discourse is predominantly ambiguous. A further discussion of implications for intercultural communication and the author's personal experience provides some insight into the continuing complexity involved in American/Japanese communication. 


\title{
THE JAPANESE/AMER ICAN INT ER FACE
}

\section{A Crosscultural Study on the}

\section{Approach to Discourse}

\section{by}

HITOMI TAMURA

A thesis submitted in partial fulfilment of the requirements for the degree of

\author{
MASTER OF ARTS \\ in \\ SPEECH COMMUNICATION
}

Portland State University 
TO THE OFFICE OF GRADUATE STUDIES AND RESEARCH:

The members of the Committee approve the thesis of Hitomi Tamura presented May 16, 1983.

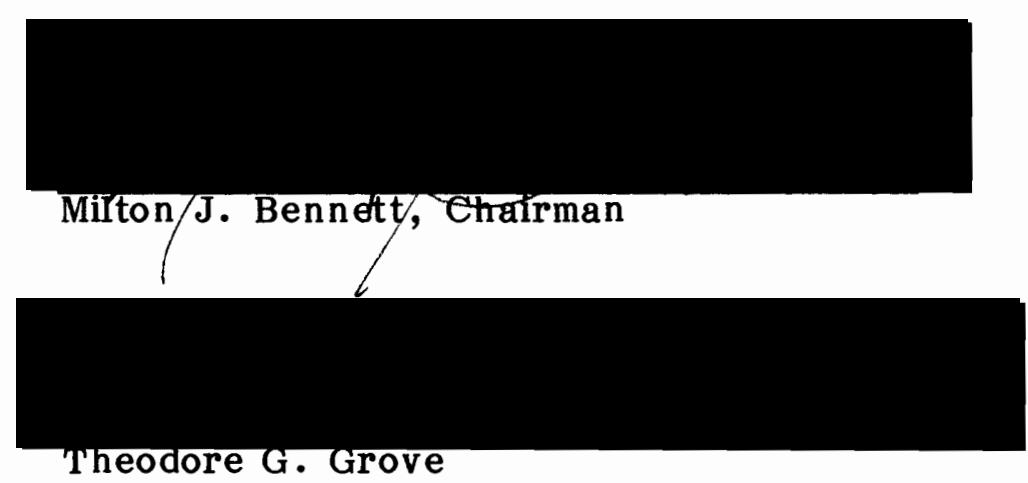

Theodore G. Grove

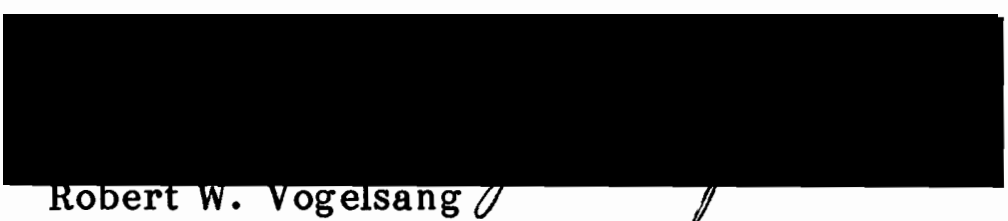

APPROVED:

Theodore G. Grove, Head, Department of Speech Communication

Stanley E. Kauch, Dean or Graduate studies and Research 


\section{ACKNOWLEDG MENTS}

My foremost acknowledgment of thanks and appreciation is to my committee members for their valuable assistance, Chairman of the committee, Milton J. Bennett Ph.D., Theodore G. Grove Ph.D., and Robert W. Vogelsang Ph.D. My long-time committee chairman, LaRay M. Barna, who was on sabbatical at the completion of this thesis, deserves special gratitude for her loving guidance. F. Gibson, S. Kosokoff, Ph.D.s and Mrs. Morrell helped me develop this study.

My special thanks are due to Ernst $\mathrm{H}$. Laemmert, who encouraged my commitment to complete this study, shared his precious experience and insight and took time out from a busy schedule to patiently review my draft.

I am grateful for the encouragement of my friends, Yvonne, Noriko, Kevin, Eiko, Steve, Kris, Rosalie, Duncan, Hiro, and Reiko, through whom I have learned a greater appreciation for the importance of communication. Yasukuni, at the University of Washington, was helpful in collecting my samples.

My grateful thanks go to my friend Bill, who made it possible for me to produce this paper with the technology he made available to me.

Finally, I wish to express my deepest love and gratitude to my father, my late mother, and my sisters who continued to support my endeavor in word and deed throughout the years I have spent away from my family. 
TABLE OF CONTENTS

PAGE

ACKNOWLEDGMENTS $\ldots \ldots \ldots$ iii

LIST OF TABLES ............................. vi

LIST OF FIGURES ........................ ix

\section{CHAPTER}

I INTRODUCTION $\ldots \ldots \ldots \ldots \ldots$

Background .................... 2

Purpose of the study .......... 5

II LITERATURE REVIEW ................. 8

Linear vs. Nonlinear Presentation ..... 8

Inductive vs. Deductive Reasoning ..... 16

. Explicit vs. Implicit Communication ..... 20

Analytical vs. Emotional Statements ..... 27

III METHODS $\ldots \ldots \ldots \ldots \ldots$

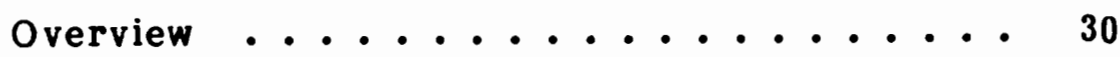

Operational Definition $\ldots \ldots . . . . .32$

Sampling Procedure $\ldots . . . . . . .36$

Selection of Raters and Subsamples ..... 38

Reliability Test ............ 40

Data Analysis .............. 42

IV RESULTS AND DISCUSSION $\ldots \ldots \ldots 45$ 
Results .................... 45

Discussion and Implication

for Intercultural Communication $\ldots . . .70$

Limitation of the study ......... 78

Suggestion for Future Research ....... 79

v PERSONAL EXPERIENCES $\ldots \ldots \ldots$

BIBLIOGRAPHY ..................... 86

APPENDICES $\ldots \ldots \ldots \ldots . \ldots \ldots$

A Instructions for the Raters .......... 92

B Summary of Coded Samples per Individual Rater ........... 97 


\section{LIST OF TABLES}

T ABLE

PAGE

I Organization of Rater and Sample

Population .............. 39

II Inter-Rater Agreement in Analysis of

Separate Subsamples ............. 4 41

III Inter-Rater Agreement in Analysis of

Twelve Identical Bicultural Subsamples .....

IV Organization of Supplementary Test for

Crossculture and Within-Culture Differences .

V Linearity of Presentation: Observed Frequencies

and Chi-Square Computation

(Results of Rater 1) ............ 46

VI Linearity of Presentation: Observed Frequencies and Chi-Square Computation

(Results of Raters 2 through 7) ........ 47

VII Directness of Introduction: Observed Frequencies and Chi-Square Computation

(Results of Rater 1) ............

VIII Directness of Introduction: Observed Frequencies and Chi-Square Computation

(Results of Raters 2 through 7) .......

IX Repetitiveness of Conclusion: Observed Frequencies and Chi-Square Computation

(Results of Rater 1) .............

X Repetitiveness of Conclusion: Observed Frequencies and Chi-Square Computation

(Results of Raters 2 through 7) ........ 51

XI Type of Reasoning: Observed Frequencies and Chi-Square Computation

(Results of Rater 1) ............

XII Type of Reasoning: Observed Frequencies

and Chi-Square Computation

(Results of Raters 2 through 7 ) ........ 
XIII Explicitness of Conclusions: Observed Frequencies and Chi-Square Computation

(Results of Rater 1) ........... 54

XIV Explicitness of Conclusions: Observed Frequencies and Computation of Fisher Exact Probability - 55 (Results of Raters 2 through 7)

XV Concreteness of Statements: Observed Frequencies and Chi-S quare Computation

(Results of Rater 1) ...........

XVI Concreteness of Statements: Observed Frequencies and Chi-Square Computation

(Results of Raters 2 through 7 ) ....... 57

XVII Width of Perspective: Observed Frequencies and Chi-Square Computation

(Results of Rater 1) ..........

XVIII

Width of Perspective: Observed Frequencies and Chi-Square Computation

(Results of Raters 2 through 7 ) .......

XIX

Definiteness of Qualifiers: Observed Frequencies and Chi-Square Computation

(Results of Rater 1) ...........

XX Definiteness of Qualifiers: Observed Frequencies and Chi-Square Computation

(Results of Raters 2 through 7 ) .......

XXI Objectivity of Evidence: Observed Frequencies and Chi-Square Computation

(Results of Rater 1) ...........

XXI I Objectivity of Evidence: Observed Frequencies and Chi-Square Computation

(Results of Raters 2 th rough 7) .......

XXIII Analyticity of Statements: Observed Frequencies and Chi-Square Computation (Results of Rater 1) ........... 64

XXIV Analyticity of Statements: Observed Frequencies and Chi-Square Computation

(Results of Raters 2 through 7 ) ....... 65

XXV Summarized Tabulation of the Ten Hy poth eses Tested ........... 66 
viii

PAGE

XXVII

Letters Analysis ............. 68

XXVIII Within-Culture Analysis:

Editorials vs. Letters ........... 69 


\section{LIST OF FIGURES}

FIGURE

PAGE

1. Rating Scale Example ............... 40 


\section{CHAPTER I}

\section{INTRODUCTION}

Today, in a world of sophisticated technology that frequently stretches beyond what would have once been considered our "wildest imagination", we of ten still find ourselves confined by a prison of our own making: the linguistic, sociological, psychological and philosophical thought structures that our own culture has imposed upon us and which we are of ten unable or unwilling to transcend.

Ever since the first time when human beings learned that there were other cultures in the world beside their own, great efforts have been undertaken to impose one's own culture onto foreign cultures, usually as a byproduct of an occupation or equivalent circumstances. This was then a result and cause of war and conflict, as it is now. In a world of complex interdependencies, people and nations are still fighting each other for essentially the same reasons (e.g. Iran/Iraq, Israel/Arabs, white South Africa/vs. its black majority etc.), even though they cannot rationally afford fighting with one another any longer. Assimilation has not been possible and seems unlikely ever to occur. So there is no hope for peace without the understanding of and appreciation of the diversity of human cultures, including languages, history, ideologies and religions. Hall (1977) urges that "the future depends on man's transcending the limits of individual cultures" (p. 2).

International negotiations concerning peace treaties, trade 
agreements, academic exchanges and tourism, are rapidly increasing in number. For Americans, the majority of international encounters of the past were with European nations and Russia due to their relative similarity of background. Today, however, quite different cultures have emerged onto the international scene, such as the oil-rich Arab nations, developing nations such as China, and African and Latin American countries.

Among the "newer" countries is industrialized Japan, whose relationship with the United States goes back in history as much as 130 years ago to the time when Commodore Perry first visited there. These two countries have become each other's foremost trading partner despite conflicts, wars, and frequent misinterpretations of the other's interests, methods and degree of commitment.

Japan is the first country in Asia to become a strong economic partner with the U.S. This has happened in spite of language and other communication difficulties. In the initial encounter, neither party had any knowldege of the spoken language of the other. Today, any political or business negotiations involve interpreters or translators. These interpreters must not only be fluent in two languages but they must consider other important factors, such as differences in nonverbal behavior, social values, perception, and belief systems. Of particular interest to this study is the need to consider differences in reasoning patterns as evidenced by their respective styles of argument.

\section{BACKGROUND}

Historically, the early civilization among Indo-Europeans was based 
on a nomadic life style mainly sustained by hunting. They were in constant contact with nature and occasionally with other tribes. This confrontation with nature and other human beings led people to draw a clear boundary around each individual and his philosophy of existence (Nakane. 1972, Nakamura. 1974).

Americans follow this aforementioned tradition and value the selfexpression of the individual in a modern society. As Ewbank (1951) states, "the discussion and debate are not simply courses in a college curriculum, they are essential tools of a democratic society" (p.3). In this context, the value and goal of arguments are to achieve a democracy. Equality encourages difference of opinion and expression of one's opinion to persuade others. Through a survey of interpersonal accessibility among Americans and Japanese, Barnlund (1975) defines American distinctiveness of interpersonal communication as follows:

In the United States and in most Western culture, the psychological unit is the solitary human being. The individual is the measure of all things. Identity must be claimed and defended by each person for himself. Integrity---the sense of inner harmony and wholeness---resides within the ego of each separate member of society. To preserve this sense of personal uniqueness and personal identity, the individual must of ten stand apart or even stand against other members of his family, of fice, neighborhood, or nation ( $p .154)$.

As a consequence of the search for equality together with a distinctiveness of self-identity, American communicative behavior is characterized by a preference for clear verbal communication. People in America learn skills of verbal communication in a variety of ways, such as argumentation, debate, discussion, persuasion, public speaking etc., in order to express their thoughts in a cohesive manner.

In contrast, Japanese society was developed from small farming communities. Having given up their original nomadic lives, the Japanese 
settled down to cultivate rice fields in one place. In an agrarian environment, families continued generation upon generation, until the members of the community knew each other so well that society as a whole became very close-knit and similar to a family. Nakamura (1974) thinks that:

....in such a society, individuals are closely bound to each other and they form an exclusive social nexus. Here an individual who asserts himself will hurt the feelings of others and thereby do harm to himself as well (p.413).

Differences in opinion of each individual are neither clearly articulated nor likely to be internally formulated. To compound this attitude, it is quite possible that the unavoidable exposure to the typical Asian monsoon weather also compelled the Japanese to adapt a passively harmonious attitude toward nature.

The feudal system, in addition to the natural environment and cohesive social attitudes, had existed in Japan until 1850 and determined the role of communication in a society very different from its American counterpart. The hierarchical concepts start within the family and are woven throughout all spheres and strata of society. Without the concept of equality, a strong awareness and expression of self is not perceptibly encouraged. Thus the Japanese learned to extend and dissolve their identity boundaries into those of others, and nature as well, a process which Nakane (1972) terms "renzoku", the concept of social and environmental continuum (p.138).

Compared to the concept of integrity valued in American society, the Japanese of ten make a distinction between "tatemae" (a principle, an of ficial stance) and "honne" (one's real intention or motive). Typically, Japanese interpersonal communication may be defined as a preference to 
"honne," the emotional self over "tatemae," the logical self. The expression of tatemae is sort of a ritual. Reality exists somewhat removed from tatemae for the Japanese. Exchanging honne between individuals causes psychological dependence upon one another and further brings about a loss of personal identity and an equivalent strengthening of the common denominator.

As a result, the communicative style among the Japanese, seeking harmony with nature in general and society in specific, is characterized by its lack of clear verbal exchanges. Thus, neither speech courses nor formal composition are taught in academic institutions. Today, only three universities in Japan of fer speech or communication theory courses in either the English or the Linguistic Department (Kawashima and Oxford, 1970. p.127). When taught, communication theory is usually just presented as a part of an English-as-a-second-language program, instead of a system aimed at clarity of verbal communication based on the assumption that people, although similar, are dissimilar in their expression of thought.

\section{PURPOSE OF THE STUDY}

The major purpose of this study is to contrast and compare American and Japanese argumentative styles as well as to illustrate a new level of awareness that individuals in both countries need to develop in order to understand each other clearly. It is believed that cultural variables have effects on the style of argument in both America and Japan. The author will examine and present the nature and theory behind the argumentative styles of both America and Japan. This will be 
done through a comparison of studies conducted in the past and a content analysis of current newspaper editorials and Letters to the Editor from both countries. Results of this study and their implications for Japanese/American communication will be presented. Finally, the difference in argumentative style explored in the thesis will be exemplified by the personal experience of the author, who is a Japanese, living in the United States and attempting to write her thesis using the American style of arg ument.

The following hypotheses are made:

1. The presentation of American arguments tends to be more linear, whereas that of Japanese arg uments tends to be more nonlinear.

2. American arguments tend to be more direct in their introduction of the subject matter than Japanese counterparts.

3. In an American argument, there is less restatement of the same conclusion than in its Japanese counterpart.

4. American arguments tend to be more inductive or deductive than their Japanese counterparts.

5. In American arguments, conclusions are more of ten explicitly stated than those in Japanese arguments.

6. Statements in American arguments tend to be more concrete than abstract, whereas statements in Japanese arguments tend to be more abstract than concrete.

7. Evidence in American arguments covers a wider perspective than that in Japanese arguments.

8. Definite and strengthening qualifiers appear more frequently in statements of American arguments than in their Japanese counterparts, where conditional and weakening qualifiers are found 
more frequently.

9. Evidence in American arguments tends to be more objective than that of Japanese counterparts, whereas evidence in Japanese arg uments tends to be more subjective than in American counterparts.

10. American arguments tend to include more analytical statements, whereas Japanese arguments have more emotional statements. This study will at tempt to contribute to a greater and more comprehensive understanding between Japanese and American people by assisting both to become aware of the differences in their respective argumentative behavior so that the communication process itself does not create additional barriers to their understanding of each other. Thus, the differences between the American and the Japanese styles of argument are not treated in terms of which communicative behavior is better or worse, but rather what mode of communication may best serve both toward a dialog ue of greater effectiveness. The goal is a better understanding and appreciation of alternative styles of argumentative behavior. The next step would be to improve ways of working together, taking these differences into account. 


\section{CHAPTER II}

\section{LITERATURE REVIEW}

A search of the literature regarding argumentative styles reveals studies from many areas, including linguistics, philosophy, English-asa-Second-Language programs, psychology, sociology and speech communication. These will be categorized according to whether they discussed the following:

(1) linear vs. nonlinear characteristics of the communication within cultures.

(2) inductive vs. deductive approaches to reasoning.

(3) explicit vs. implicit communication forms.

(4) analytical, objective vs. emotional, subjective sta tements.

These terms will first be defined, followed by a review of the literature in each area.

\section{LINEAR VS. NONLINEAR PRESENTATION}

In this section, both American and Japanese argumentative styles are examined in terms of their forms, either as a linear presentation or as a nonlinear presentation. A linear presentation requires a logical development within a paragraph and appropriate use of conjunctions. A nonlinear presentation, in contrast, does not require logical linkage 
within a paragraph and seldom has conjunctions.

A linear presentation is here defined as a chain-like organization which consists of a succession of steps that are dependent upon the previous supposition. Each step is linked logically and the process itself is of vital importance. Therefore the decoder, or audience, is able to follow the line-like process of the encoder's thoughts.

A nonlinear presentation is considered to be a point-like organization which consists of statements that are neither related internally nor dependent upon the progression of statements. Since the encoder organizes his thought in a seemingly random manner, the decoder is supposed to supply what is left unstated, and then to interpret the encoder's thought.

Several authors compare the Japanese nonlinear form of discourse to a Japanese art form, the Haiku, which is a form of poetry written in seventeen syllables (Oide 1965, Inukai 1966, Kunihiro 1973). To exemplify this comparison and to act as further illustration of a nonlinear presentation, the following haiku by the classical Master Basho is offered: Hana no kumo kane wa Ueno ka Asakusa ka.

A cloud of blossoms--

a bell--

Ueno? Asakusa?

(Henderson, 1958 p.4)

In this haiku, Basho outlines important parts such as spring, dusk, Tokyo downtown spots, and a temple bell and readers must fill the rest for themselves. The mind of the reader is intrigued and stimulated by the apparent and intentional lack of conjunction between the items strung 
together without an obvious link, like pearls of different color, size and luster threaded together. This way, the imagination of the decoder may come to any of an almost endless combination of meanings and images-both physical and philosophical. A few examples of translation show the versatility of interpretive understanding through this highly developed art form of the unsaid--the haiku.

\author{
A vesper bell-- \\ through the cloud of cherry blossoms, \\ from Ueno--or is it Asakusa's?
}

Through a cloud of cherry blossoms

tolls a vesper bell---

is it Ueno's or that from Asakusa?

This vesper bell

like a cloud of cherry blossoms

tolls from Ueno--or is it Asakusa?

Thus, haiku, intended to be interpreted by the reader, has the same quality as the Japanese arg umentative style. According to Inukai (1966),

Although the seventeen syllables which make up the haiku have, of course, meaning simply as words, almost the whole of its effect derives, not from the words which are arranged visibly into the seventeen-syllable form, but in the emotional overtones which hover invisibly about them. This remarkable literary form is of extraordinary beauty and refinement, but it lacks entirely the Western-style rhetoric which presents things for all to see, analyzes them, then makes doubly sure that its meaning is quite clear. Haiku is not a literature of speaking one's mind. In the same way, it seems to me that the Japanese manner of arguing has haiku-like qualities, and that their ways of thought in general betray the same traits $(p .23)$. 
In American classrooms, according to Kaplan (1966), writing a coherent paragraph which is linear in its development, using either inductive or deductive reasoning, is of great importance. In contrast he investigates foreign students' expository writings, and concludes that oriental writing is particularly characterized by its indirection and circular flow of arguments.

The circles or gyres turn around the subject and show it from a variety of tangential views, but the subject is never looked at directly. Things are developed in terms of what they are not. Again, such a development in modern English paragraph would strike the English reader as awkward and unnecessarily indirect (P.10).

Kaplan regards the Eight-Legged Essay form as a main source of Chinese elaborate indirection. The Eight-Legged Essay which is the standard device in the civil service examination from the middle of the fifteenth century into the twentieth century still influences Chinese and Chineseinfluenced languages. An eight-legged Essay consists of:

1. The breaking open

2. Accepting the title f parallel with each other

3. Embarking (Introductory discourse) )

4. Introductory Corollary parallel with each other

5. First Middle Leg parallel with each other

6. Second Middle Leg )

7. First Final Leg parallel with each other

8. Tying the Knot (Kaplan, 1971. p.49) Obviously this form shows elaborated parallelism and a long introduction to the topic. Kaplan concludes that oriental writing is characterized by its circular movement of thought resulting from its indirect approach to 
the subject matter, whereas Western writing is characterized by its linearity .

In contrast to the importance Americans place on developing a coherent paragraph in their classrooms, the Japanese seldom learn the analogous paragraph organization. Toyama (1976) leads us to the conclusion that it is this lack of good paragraph development that presents difficulty to the Japanese in the course of writing a long coherent work.

Nisio (1957) observes that although itemization is of ten used in Japanese articles, each item does not interrelate with each other logically, nor is it arranged in a systematic order. In contrast, American itemization is a state where sentences outline a logical linkage among items, and where each new item may be represented by a new paragraph. Nishio explains the attitude of the Japanese, who are not conditioned to express their thoughts sequentially to make themselves understood by an American. He feels that Japanese, although they sincerely wish to express the truth, tend to be convinced that it is the very sincerity in their expression of thought or feeling that is sufficient in the persuasion of their audience. A further step to organize their thoughts in a logical sequence is deemed an unnecessary and even irrelevant one, preventing the development of this intellectual discipline.

Several linguists point out the Japanese tendency to minimize conjunctions as the means of a logical and sequential linkage between sentences or thoughts. Oide (1965) indicates that the Japanese tend to avoid using conjunctions, since the function of conjunctions is the determination of the relationship between sentences and thus would leave no room for implications. Doi (1974) also states that conjunctions are 
often used only to "cement and induce the speaker's free association... We can go on talking for hours without coming to the point" (p.23).

Johns (1979) comes to the same conclusion. She finds that the Japanese use conjunctions infrequently by her error analysis of the cohesion of business prose from five separate language groups. Johns also points out that "...rather than developing an idea through one of the standard rhetorical devices (detail, description, example) the student repeats himself in a number of ways, realizing an unnecessary redundancy" (pp.317-318).

Kunihiro (1973), an anth ropolog ist, calls the Japanese mode of argumentation a "dot-type" discourse which "is a presentation of one item after the other in a highly anecdotal or episodic vein: conclusions are seldom articulated "(p.100). A dot-type communication is possible in a society such as Japan where people share similar values, he states. Provided with some points, the reader or the audience connects and subjectively comprehends them through interpolation and interpretation. This is in contrast with the American encoder-oriented process where it is the encoder's goal to make a point and persuade the decoder. Kunihiro (1976) explains this decoder-involved process in the following manner: ... [whereas] in Japan there is a sense of leisurely throwing a ball back and forth and observing each other's response, in the language of the West, logic and reason are continuously tossed between speaker and listener, as if one's identity were constantly being questioned, and throughout, there is a sense of reinforcing each other's independence. In Japanese-speaking society, the individual and the whole are organically integrated, and as long as one is following the prescribed route, not only communication of ideas but everything else follows without disruption (p.275).

A main distinction between American and Japanese argumentative styles is thus whether or not logic plays an important role in the discourse.

Nakamura (1974) states, from the philosophical point of view, that 
Japanese are inclined to neglect $\log$ ic and emphasize the emotional factors over the cognitive factors. He points out that one of the characteristics of the Japanese way of thinking is a tendency towards the non-rational. These are broken down into: a) a tendency toward the illogical; b) a weakness in the ability to think in terms of logical (con)sequences; c) a tendency toward the intuitive and emotional; d) a lack of ability to form a complex (re)presentation; $f$ ) a weakness in the knowledge of the objective processes involved (pp. $531-576)$.

In the study of the style of Asian oratory, Oliver (1974) explains characteristics of the Asian discourse as follows:

Students of debate will note readily that the Chinese debaters neither depended upon an interweaving of logic and facts, as do most American collegians,... What they [Asian] will find is a direct clash of arguments based upon substantial philosophical premises, in which each debater seeks to make his fundamental assumptions appear to be in accord with the life experience of the listeners ( $\mathrm{p} .103)$.

Thus, the Asian method of public address is characterized by an absence of logic and as audience-involved discourse aimed toward a mutual agreement.

In contrast, Western rhetoric in the Platonic and Aristotelian tradition is a "pattern of manipulative devices or techniques by which a speaker may influence listeners" (Oliver, 1971.p.7). Therefore the Western method of discourse is to think purposely and which requires:

...a sharpening of focus, a narrowing and delimiting of the field of observation, a shutting out or diminishing of all that does not contribute to acceptance of the speaker's preconception. To think purposively means to impose upon a segment of reality the individual conclusions of the thinker (Oliver, 1974. p.107).

Thus the American public address, seeking for the truth, tends to be coherent and linear, based on logic, while Japanese discourse, promoting harmony, tends to be indirect and incoherent. It is this very contrast 
that promotes the different role of the newspaper in America and Japan. Beniger and Westney (1981), pointing out the informative and educational role of Japanese newspapers, state:

Criticism has come from several U.S. writers who note the emphasis on information rather than on advocacy... With an accompanying neglect of critical investigative journalism (p.15).

Moran (1973. p.863), a psychologist, studies the individual differences in the cognitive dictionary structure, as reflected in the free word association among American and Japanese families. His four categories can be summarized as follows:

1. Iconic transformations: a word pair which refers quality to the referent or vice versa, e.g. red-apple, beautiful-flower.

2. Enactive transformations: a word pair descriptive of action on the referent, e.g. apple-eat.

3. Functional transformations: a word pair which represents two physical referent in a functional relationship, e.g. table-chair.

4. Logical transformations: word pairs in what linguists term a "sense" relationship.
a. synonym: e.g. blossom-flower.
b. superordinate: e.g. cabbage-vegetable.
c. contrast: e.g. dark-light.
d. coordinate: e.g. apple-pear.

In general, this research finds that both American and Japanese children use primarily enactive transformations, American parents use principally logical transformations, and Japanese parents prefer mainly iconic transformations. The results indicate that the culture is the predeterminant of an adult's basic dictionary structure or word usage. 
As a result, one can observe a shift from enactive to logical transformation by American children as they grow up, and analogously a shift by Japanese children from enactive to iconic transformations during their adolescence. Thus, culture seems to be the foremost factor in perpetuating a logical discourse as a dominant mode in America and a nonlogical discourse in Japan.

In summary, American argumentation is predominantly linear, as evidenced by its preference for coherent paragraphs, sequential itemization, and its reliance on logic. Japanese argumentative style, in contrast, is predominantly nonlinear, as evidenced by its haiku-like presentation, incoherent itemization and its apparent disregard of logic.

\section{INDUCTIVE VS. DEDUCTIVE REASONING}

According to Aristotle, induction is "a passage from the individual to the universal, e.g. the argument that if the skilled pilot is the most effective, and likewise the skilled charioteer, then in general the skilled man is the best at his particular task" (Mckeon, 1941. p.198). Brembeck and Howell (1952) define induction as "the process of drawing a conclusion from the examination of specific data" (p.194) and deduction as the reverse process which "begins with the acceptance of a general statement and applies to a specific instance" (p.194). In the context of this paper, inductive reasoning is defined as reasoning which proceeds from the specific to the general and deductive reasoning as reasoning which proceeds from the general to the specific.

Kaplan (1966) indicates that English paragraphs reflecting typical thinking patterns are linear in their development and use both inductive 
and deductive reasoning:

An English expository paragraph usually begins with a topic statement, and then, by a series of subdivisions of that topic statement, each supported by example and illustrations, proceeds to develop that central idea and relate that idea to all the other ideas in the whole essay, and employ that idea in its proper relationship with other ideas... Contrarily, the English paragraph may [also] use just the reverse procedure; that is, it may state a whole series of examples and then relate those examples into a single statement at the end of the paragraph... These two types of development represent the common inductive, deductive reasoning which the English reader expects to be an integral part of any formal communication (p.4).

Condon and Yousef (1975) agree with Kaplan's view regarding

English usage. They state effective speech and writing in the AngloAmerican culture are one of the following: either a pattern starting with a generalization or a conclusion that is then followed up by its supports and details, or as an alternate pattern, a series of specific points at the outset which then come to a generalization or a conclusion. They go on to say that in contrast, Japanese speech organization is either a series of generalizations or a series of specific but unrelated points (pp.240-241).

A similar conclusion is stated by Kunihiro (1973), an anthropologist, who classifies Japanese presentations into two types. The first type is a dot-type and the second is:

...observed largely in statements made by people who may have been influenced by continental philosophers. These people tend to think deductively, presenting maxims and axioms as they are, of ten unaccompanied by actual data ( $p .100)$.

Kunihiro contrasts this statement with the observation that an American presentation usually starts with a series of concrete facts and data and leads to a general conclusion.

Kobayashi (1976) defines Japanese reasoning and concludes that the Japanese speak on the periphery of the subject first and come to the 
conclusion. Kobayashi based his conclusion on interviews of Japanese students wishing to study in France. He studied how they went about describing their purpose, interests, goals and motivations together with their current outlook and position in life, and how they subsequently came to their respective conclusions wishing to go abroad. This pattern resembles "nemawashi" (digging around the roots of a tree), that is to maneuver behind the scenes.

Pribram (1949) studies the same subject through the historical and philosophical point of view. He calls attention to the differences in the methods of reasoning or patterns of thought in the Western world. Four styles of reasoning are differentiated: the universalistic pattern in France, the Mediteranean countries and the Latin American nations; the nominalistic pattern in the Anglo-Saxon countries; the intuitional or organismic pattern in Germany and Slavic East Central Europe; and lastly the dialectical pattern of the Soviet Bloc and other Marxist countries. Nominalistic reasoning, of ten seen in American reasoning patterns, is thinking in terms of hypothetical concepts. Pribram's basic theory is that all concepts are free creations of the human mind and "knowledge and understanding of phenomena were held to be attainable primarily by experience" (p.11). Thus nominalistic reasoning is tied with empiricism and its reasoning which proceeds from the particular to the general, i. e. it is characterized by induction. Pribram makes reference to the Japanese pattern of thought and classifies it as an oriental universalistic reasoning. In universalistic reasoning, "reason is credited with the power to know the truth with the aid of given general concepts and to establish absolutely valid rules for the organization of human relationships in accordance with these concepts" (P.7). Universalistic reasoning, 
therefore, proceeds from the general to the particular, that is, deduction. The other two styles of reasoning (organismic and dialectic) are not considered since both are outside the constraints of this discussion.

Stewart (1974), who compares American modes of thinking with European counterparts, concludes that foreign students' difficulties in writing efficient term papers and dissertations are principally due to the different styles of thinking. He states that, "...for Americans, the world is composed of facts--not ideas. Their process of thinking is generally inductive, beginning with facts and proceeding to ideas" (p.16). Stewart thus points out the preference of inductive reasoning in the American academic field of endeavor.

The analysis of the different cultural styles of persuasion is introduced by Glenn, Witmeyer and Stevenson (1977). They investigate United Nations Security Council manuscripts of American, Soviet and Arab countries based on the assumption that:

...the negotiators select approaches consistent with their own past experiences and within the cultures to which they belong, and that they are selected, in part, on the basis of their ability to handle a style congruent with the culture" (p.53).

They contrast three different styles: the Factual-Inductive, the Axiomatic-Deductive and the Affective-Intuitive, and state that the combination and dominance of the three styles determine the cultural style of persuasion. They conclude that the American mode of persuasion is dominated by the Factual-Inductive style, and at tribute the AxiomaticDeductive and Affective-Intuitive styles to the Soviet Bloc and Arab countries, respectively.

In summary, previous studies by linguists, philosophers and 
communication scholars suggest that Americans generally use a linear organization for their speech and written communication, using either inductive or deductive reasoning. Japanese organize their verbal and written discourse by supplying to the audience either a series of related or unrelated generalizations or specific points which are like dots and points. Yet another study shows that Japanese tend to use deductive reasoning. Thus the consensus of the reviewed scholars defines the American style as linear and inductive/deductive as compared to the Japanese style as being nonlinear and neither inductive nor deductive.

\section{EXPLICIT VS. IMPLICIT COMMUNICATION}

This section treats the argumentative styles of American and Japanese in terms of explicit communication vs. implicit communication. Explicit communication consists of the precise use of language, exact quotations, enhancing qualifiers, and overstatements. Implicit communication consists of implied and ambiguous use of language, fondness for weakening qualifiers and understatement.

In the first part of this section, American and Japanese argumentative styles are examined, contrasting the concepts of explicit vs. implicit communication. In the second part of this section, several modes of communication by various scholars are introduced and then are examined to make a clear distinction between American and Japanese styles of argument.

In a classic writing manual, The Elements of Style, Strunk and White (1979) recommend to "use definite, specific, concrete language" (p.21). Tanizaki (1975), a Japanese leading novelist, affirms the clear 
use of words in English and ambiguous use of words in Japanese. In

Bunsho Tokuhon [A literary Composition Reader], Tanizaki compares the manuscripts of Tale of Genji by Murasaki Shikibu with its English

translation by Arthur Waley:

The English, in short, is more precise than the original; nothing is indistinct in meaning. In the original, parts that can be understood or left unsaid are as a rule left unsaid; in the English even what is evident is made more evident... Clarity is indeed gained when, as in the English, everything is there to be seen, but meaning becomes so much the more circumscribed and shallow... Japanese literati therefore avoid such a futile endeavor and intentionally use expansive words, such as those sensuous elements as style, rhythm, and the appearance of the characters. This is why I have written that in classical Japanese literature each word is surrounded by shadows and hidden implications like the corona around the moon. In other words, a spare use of words becomes an illusion beckoning the imagination of the reader to fill in what is lacking ( $p .56)$.

In the analysis of the Japanese communicative style from a judicial point of view, Kawashima (1979) stresses that the lack of clarity in Japanese words results in various judicial interpretations of statutes. Although Japanese jurisprudence is based on Western models, Japanese law deals in quite amorphous terminology. By contrast, Westerners use vocabulary in a more precise and specific manner. Japanese words have core meanings but their peripheral meanings are vague. Kawashima (1975) states the historical lack of the concept of equality and independence of individuals as one of the major obstacles to clear communication. He explains,

...in such a culture [Japanese], the social order consists of social obligations, which are defined, not in specific, determinate terms, but in diffuse, indeterminate terms, and the indeterminateness of social obligations of this nature contains the possibility of flexible adjustment to contingent circumstances. The indeterminateness of individuals... does not allow the existence of the concept of "right" [and wrong] as the counterpart of social obligations (p.274).

American fondness for contracts also contributes to the precise use 
of their language. Van Zandt (1976) states that "Westerners usually want the contract to indicate commitments, conditions, and restrictions in precise language which will not be liable to later misinterpretation" (p.317). The Japanese attitude toward contracts is different from that of Westerners. The Japanese tend to draw up a contract as a written agreement concerning the expression of their intention and that business association in general. Kawashima (1975) explains the Japanese attitude toward disputes:

In the world of business, people in Japan traditionally avoid defining the contractual parties, not to mention actual negotiations on the terms for eventual resolution of disputes, would indicate a mistrust of the other party to the contract and would damage the relationship of wa (harmony), the most valid reason, in the minds of contractual parties, for compliance with the obligations assumed under the contract. Therefore, contracts were rarely documented (p.266).

In short, Americans favor the explicit style of argumentation as evidenced in contract. In contrast, Japanese will try to sense the other party's feelings and act on that basis without having to draw up contracts.

The typical American interest in exact quotation is pointed out by Condon and Yousef (1975). For example, in order to be precise and objective, American editors may request their correspondents in Japan to send exact quotes of the politician's speech that they are to cover. One of these correspondents complains as follows:

If I quote what he actually said, it rambles, it seems vague, it is totally unquotable. Yet if I rewrite all that to indicate what everybody here knows he meant, then I am putting words in his mouth that he never would have said ( $p .239)$.

Studies indicate that qualifiers are used more of ten in Japanese than in English. Mehrabian (1971) asserts that people use weakening qualifiers in order to minimize responsibilities (p.97). As a result of the 
high value and the emphasis Japanese society puts on harmony, Japanese people dislike to contradict or to be contradicted. Thus qualifiers play an important role in the Japanese language. Suzuki (1975), a linguist, states that in translating Japanese scholarly works into English, it is almost impossible to translate the frequently used Japanese qualifiers (p.27). Condon and Yousef (1975) agree with Suzuki and state that "maybe," "perhaps," "probably," and "somewhat" are frequently used by the Japanese (p.217). In contrast, Strunk and White (1979) discourage English writers from using weakening qualifiers, saying, "statements qualified with unnecessary auxiliaries or conditionals sound irresolute" (p.20).

Okabe (1981) states that Americans tend to use overstatement, oversimplification and exaggeration whereas Japanese tend to use understatement and implication (p.26). Norbeck and DeVos (1972), through their survey of projective tests, conclude that:

A continuing weakness of Japanese scholars employing projective tests has been a general reluctance to interpret the findings of the tests. When an interpretation is made, the basis for the conclusions represented is seldom clearly stated (p.30).

Authors in various fields have at tempted to model communication based on the explicit/implicit difference examined above. For instance, Mushakoji (1976), a political scientist, presents a framework that compares the Japanese-American style of communication in terms similar to those discussed above as "implicit" and "explicit". He observes the difference in the way people see themselves in relation to their physical and social environments and then terms the American way as "erabi" (selective) and Japanese way as "awase" (adjustive). The erabi view is an active mode in which human beings can manipulate their social as well 
as natural environment for their own purposes. The speaker constructs his messages for the purpose of persuading the audience. Mushakoji explains that:

...the erabi view also implies a logical structure composed of concepts and their opposites : hot versus cold... Thus, the erabi is the $\log$ ic of choosing the best one from a set of alternatives composed of selected factors from various dichotomies ( $p .40)$.

The awase view is a passive mode in which human beings adjust themselves to the physical and social environment. According to the awase view,

...the environment is not characterized by dichotomous concepts, such as hot versus cold. Rather, it may be somewhat hot or somewhat cold. Awase is the logic of seeking to apprehend [grasp] and adapt to these fine graduations of change $(\mathrm{p} .40)$.

... in contrast to the standardization of vocabulary in erabi societies, awase logic does not depend upon standardized word meanings. Expressions have multifarious nuances and are considered to be only signals which hint at reality rather than describing it precisely. Words are not taken at face value; it is trusted most and one is expected to act on it, in awase society, it is possible to"hear one and understand ten". It is interesting to note that in Japan it is considered virtuous to "catch on quickly" (sasshi ga hayai), in other words, to adjust to someone's position before it is logically and clearly enunciated ( $p .43)$.

American arg umentative style can be characterized as an explicit erabi pattern. In contrast, Japanese style is an implicit awase pattern. The second framework is introduced from an anthropological point of view. Hall (1977) discusses the importance of the role of societal context. "High context" communication is one in which most of the information is either in the physical context or internalized in the person, and very little is in the expressed message. In contrast, a "low context" communication is one in which much of the information is expressed in the explicit code and very little is implied. Hall sees the American style of communication as generally low context whereas Japanese tends to be high 
context. The Japanese in their high context societal communication imply much and take much insight for granted and therefore tend not to be specific. He feels this could account for the way Japanese "talk around the point" in the view of an American. Thus a high context Japanese communication represents an implicit style of arg ument, while a low context American communication represents an explicit style of arg ument.

Bernstein (1964), a sociolinguist, discusses the interrelationships between social classes and their coding systems. "Elaborated codes" are a person-oriented speech system in which the speaker relies on the verbal elaboration of his experiences and in which the listener is subsequently dependent upon the verbal elaboration of meaning as well:

As a child learns an elaborated code,... He comes to perceive language as a set of theoretical possibilities for the presentation of $h$ is discrete experience to others. An elaborated code through its regulation induces developmentally in its speakers an expectation of separateness and difference from others $(p .65)$.

An individualistic, person-oriented society, such as America, can be seen as having an elaborated communication system.

In contrast, in a culture where members share common life-styles and consciousness, verbal elaboration of ten becomes unnecessary. People use "restricted codes" defined as a social status-oriented speech system. Bernstein outlines the characteristics of the restricted code as follows:

The burden of changes in meaning would be carried through the extraverbal component of the communication. The "how" of the communication would be important rather than the "what". The discrete intent of the speakers, the "I" of the speakers, would be transmitted not through varying their verbal selections, but through varying the expressive features of the communication, through changes in gesture, physical set, intonation, facial modification... The speech sequence... would tend to be dislocated... disjunctive. There might well be logical gaps in the flow of meaning. The speakers would not be worried because they could take much for granted. The connecting devices in the speech might not clarify the 
logical organization of meaning. In fact, the observer might find that the meanings were strung together rather like beads on a string rather than being logically ordered (pp.61-62).

Therefore an elaborated speech system appears to be similar to erabi or low context communication style representing the style prevalent in the American argument. The Japanese argumentative style is represented by restricted codes, awase and high context.

From the psychological point of view, Deikman (1973) and Ornstein (1973) offer another way to look at the different strategy in argumentation between America and Japan. They present two modes of thought processes: 1.linear, analytical Western thought process and 2. relational, holistic Eastern thought process. Deikman describes two major modes of viewing the world as the "action mode" and the "receptive moden. An action mode is a state which manipulates the environment to achieve personal goals. "Focal attention, object-based logic, heightened boundary perception and muscle controls over the sensory system" (p.68) are the major characteristics of the action mode. Deikman defines the receptive mode as one which attends to the environment. The sensoryperceptual complex characterizes the receptive mode that dominates the muscle system and the paralogical thought process which in turn reduces boundary perception. These two modes of consciousness exist simultaneously in man and the dominant mode results in each individual's different strategy for engaging the world, with the mode choice being determined by the culture. Deikman notes,

In Western Civilization, the orientation is toward the individual's exerting direct voluntary control over all phases of his life. This orientation of control is enhanced by the ideal of the self-made man and by the pursuit of material and social goals-- all of which call for manipulation of the environment and of the self. The action mode dominates our (Western) consciousness ( $p .71$ ). 
In contrast, Eastern consciousness ap pears to be dominated by the receptive mode.

Summarizing the works by Gaggamiga (1973) and Bogen (1973), Ornstein (1973) suggests that man's left cerebral hemisphere of the brain is predominantly involved with analytical, logical thinking. In contrast, the right hemisphere is more holistically and relationally oriented. This would indicate that the Oriental is predominant in the use of his right hemisphere as compared to the left hemisphere predominance of the Occidental.

Even though four models of communication have been developed in different fields---political science, anthropology, linguistics and psychology, they reach similar conclusions. The American argumentative style predominantly exemplifies the erabi, low context, elaborated and left hemisphere communication that is explicit. The Japanese arg umentative style, in contrast, commonly typifies awase, high context, restricted, and right hemisphere communication that is implicit.

ANALYTICAL, OBJECTIVE VS. EMOTIONAL, SUBJECTIVE STATEMENTS

This section treats the subject in terms of viewing analytical and objective statements vs. emotional and subjective statements in arg uments. This juxtaposition generally overlaps with the previous two categories, linear vs. nonlinear and explicit vs. implicit, mainly due to the fact that such analytical-objective or emotional-subjective evidence in the argument is a consequence of the previous two categories.

Matsumoto (1975), comparing evidence from both American and Japanese essays, concludes that evidence used in an American essay is 
generally objective and specific, whereas apparent evidence in Japanese essays is of ten not included, or if there is stated evidence, it tends to be very general and based on the author's feeling. The assumption that the writer subconsciously depends on the reader's interpretation results in an inspecific and nonanalytical manner of writing. Kobayashi (1976) supports Matsumoto's view, stating "...in Japanese reasoning, individual interest and motivation are of ten mentioned as a matter of course" $(\mathrm{p} \cdot 143)$.

To sum up, the author found Americans to present their argument with as much detailed (analytical) and factual (objective) support as possible, with the underlying supposition that anyone, regardless of his background, will come to the same conclusion with the given data as the encoder did. The Japanese, in contrast, will typically persuade in using a personal, emotional and subjective approach appealing to the empathy, even sympathy, of the decoder.

The review of the previous studies has included research from a number of fields. First, works on linear vs. nonlinear communication of American and Japanese cultures were discussed. Past studies concluded that the Americans use a linear form of argument in its development, whereas the Japanese use a nonlinear form. It was noted that Japanese argumentative style resembles the haiku style which provides very few points of information to the reader, thereby by necessity stimulating his imagination.

The second part of the review dealt with the studies on inductive vs. deductive reasoning. The American argumentation follows either inductive reasoning or deductive reasoning. Some studies indicate that 
inductive reasoning is more frequently used than deductive in American arguments. The Japanese argument, in contrast, does not follow linear development but rather presents either unrelated points or deductive reasoning together with axiomatically accepted " $\operatorname{truth}(s) "$.

The third part of the review discussed various modes of communication and showed the explicit nature of the American argumentative style and the implicit nature of the Japanese argument.

The fourth part of the review overlapped largely with the first and third categories. Evidence used in American argument is predominantly analytical and objective in nature. In contrast, evidence in Japanese argument is principally emotional and subjective in nature.

Through this brief literature review it became apparent that a general consensus exists as to the specific differences between Japanese and Americans in the style and approach to argument. Surprisingly, although numerous studies have been conducted in various related fields, lit tle empirical research on differences in the style of argument exists. Thus the following chapter will deal with statistical methods applied in order to empirically and impartially validate or reject the crosscultural differences that were discussed earlier. 


\section{CHAPTER III}

METHOD

\section{OVERVIEW}

The preceding chapter briefly surveyed the past studies regarding the argumentative styles in both America and Japan. The focus of this chapter will be on the content analysis of current newspaper articles as a reflection of culturally conditioned characteristics, strictly in regard to the manner in which people from the two groups organize their thoughts in writing. Content analysis is defined as "a research technique for making replicable and valid inferences from data to their context" (Krippendorff, 1980. p.21).

Krippendorf f further indicates that:

... it is a fundamental fact of human communication that language is used to convey knowledge and to understand the knowledge conveyed by others. Therefore, one task of content analysis is to achieve this kind of understanding of linguistic data and to draw inferences on the basis of this understanding (p. 43).

Samovar and Porter (1982) suggest that "verbal messages consist of spoken or written words (speaking and writing are word-producing behaviors)"(p.27). Specifically this study is focused upon culturally influenced uses of language and styles of argument. Samovar and Porter add that:

Culture shapes the individual communicator. It largely is responsible for the entire repertory of communicative behaviors and meanings each person possesses (p.32).

Assuming this culturally influenced world view, the communicative or 
argumentative behavior through language could be studied in terms of either written or spoken discourse.

In oral communication,

...the verbal message is only one of many messages being transmitted to members of the audience. There are also messages in tone of voice, facial expression, and bodily action. Each of these messages tends to stimulate meaning (McCroskey, 1968. p p. 206-207).

Thus the relative importance of nonverbal communication is one of several variables that would have to be studied as a part of the total message. Without denying the importance of oral and nonverbal modes of communication, this study assumes that basic culturally conditioned organizational patterns will be reflected in written messages, and it assumes that understanding differences in these cultural patterns will contribute to improved intercultural communication.

Among written discourse, newspaper was selected as the medium because of its wide readership and the availability of materials from both cultures. Letters to the editor and editorials were further selected to illustrate the typical styles of both Americans and Japanese. Letters to the editor were thought to represent best a cross section of argumentation style of the population, whereas the editorials would best represent the educated professional stating his point with eloquence.

The selected samples were then rated by the author and nine outside raters independently according to the ten variables. In order to measure the extent of agreement among ten raters, rater reliability was assessed by using percentage of the agreement achieved between raters. The results of six raters, three Americans and three Japanese, who coded six distinct subsamples selected at random from their respective newspapers in regard to ten variables, were used as control and compared 
with the results of rater 1 , the author. The rating results of a set of twelve bicultural subsamples in randomized order judged by four raters, including the author, were compared against one another in order to test for inter-rater reliability. (Further details are found under the section describing the reliability test.)

Since the measurement involves the quantification of observations in discrete categories, the statistical model used for hypotheses testing was either a Chi-Square or a Fisher Exact Probability test, depending upon the sample size and number of categories. Computed values were based on the relative frequencies in each category rated by the author and six outside raters.

\section{OPERATIONAL DEFINITION}

For the scope of this paper, the terms "argument," "discourse," and "rhetoric" have not been distinguished semantically, even though the author is fully aware of the different connotations inherent in the terms. But since it is the purpose of this study to examine obstacles to crosscultural communication between American and Japanese communicators, a narrow semantic distinction between aforementioned terms was deemed irrelevant within the context of this paper. However, since the author is using the term argument with its derivative forms throughout the study, it may be helpful to the reader to insert the following general definitions by a number of scholars. Accordingly, the term argument can be defined as:

the sequence of interlinked claims and reasons that between them, establish the content and force of the position for which a particular speaker is arguing (Toulmin, 1979. p.13).

the establishing of reasonable connections and relationships 
between and among ideas. Argument is the logical movement from fact to fact, from facts to inference, from inference to inference, and sometimes from inference to judgments or conclusions on still higher levels. Argument is also the connecting and relating of what we believe we know (in the form of conclusions and accepted principles) to specific events and instances (Nadeau, 1972. P.44).

a particular part of a message which is designed to gain acceptance of a particular conclusion (McCroskey, 1968. p.77).

to influence those about us either through the spoken or written word... The real process of arg ument is the process of demonstrating conclusions from facts or premises that have been established as truths. It should be remembered the goal of argument is influencing attitudes and is not exhibitionism; it is to influence conduct and not just to engage in a dispute (Huber, 1963. p.4).

The hypotheses and their definitions are outlined below and the definitions are those provided to the raters.

Hypothesis \#1: The presentation of American arguments tend to be more linear whereas Japanese arguments tend to be more nonlinear. A linear presentation is defined here as a succession of steps that are dependent upon the previous supposition. A nonlinear presentation consists of statements that are unrelated internally or independent of the progression of statements.

Hypothesis \#2: American arguments tend to be more direct in their introduction of the subject matter than Japanese counterparts.

The relative direct introduction is defined as dealing with the subject matter from the first paragraph (if the topic is about a matter previously discussed, the explanation about the discussed topic is not counted). The relative indirect 
introduction is defined as a rhetorical style of using introductory paragraphs that do not concern the immediate subject matter, but of fer extraneous background arguments or lead-ins.

Hypothesis \#3: In an American argument there is less restatement of the same conclusion than in its Japanese counterpart.

This is defined as the relative degree of repetitiveness with which an argument or its conclusions are presented.

Hypothesis \#4: American arguments tend to be more inductive or deductive than their Japanese counterparts.

Inductive reasoning is defined as reasoning which proceeds from the particular case or cases to the general rule. Inductive reasoning, therefore, is aimed at establishing a general rule or axiomatic truth. Induction is the process of observation of facts and verification of the observation. Deductive reasoning is defined as reasoning which proceeds from the general rule to the particular case. Deduction is the process to check the implication of the hypothesis or general rule with the particular case or fact.

Hypothesis \#5: In American arguments, conclusions are more often explicitly stated than those in the Japanese arguments.

A conclusion is any belief that an encoder wishes his decoder to accept. 
Hypothesis \#6: Statements in American arguments tend to be more concrete than abstract, whereas statements in Japanese arguments tend to be more abstract than concrete.

Concrete statements are characterized by the use of the writer's immediate observable experience, therefore are mainly descriptive, specific, and particular. Abstract statements are disassociated from any specific instance and personal input and deal with the subject more theoretically.

Hypothesis \#7: Evidence in American arguments covers wider perspective than that in Japanese arguments.

A wide perspective is defined as giving a multiple evidence base from which to draw a conclusion. A narrow perspective is giving a singular or small evidence base toward the subject's conclusion.

Hypothesis \#8: Definite and strengthening qualifiers appear more of ten in statements of American arguments than in their Japanese counterparts, where conditional and weakening qualifiers are found more frequently. Definite qualifiers include "absolutely," "definitely," "certainly," "necessarily," etc. Conditional qualifiers include "presumably," "probably," "maybe," "very likely," "plausibly," "seemingly," "possibly," etc.

Hypothesis \#9: Evidence in American arguments tends to be more objective than that of Japanese counterparts, whereas evidence in Japanese arguments tends to be more subjective than American 
counterparts .

Evidence is a basis for subsequent conclusions. Criteria for subjective evidence include personal opinions, common or generally accepted knowledge, and personal feelings toward the subject matter. Criteria for objective evidence include statistical data, detailed accumulation of facts, experimental observations, previously established conclusions and personal expert testimony with a justifying underlying basis.

Hypothesis \#10: American arguments tend to include more analytical statements, whereas Japanese arguments have more emotional statements. Analytical statements are defined as logical statements which separate or break up the subject into its constituent parts and express the author's rational thoughts rather than his emotion. Emotional statements are defined as expression of the author's personal feelings which do not necessarily accompany logic.

\section{SAMPLING PROCEDURE}

In this study, three of the most reputable newspapers from America and Japan were selected. Among approximately 1750 daily newspapers and 9400 weekly newspapers, "the United States, unlike many other countries, has no truly national newspaper" (Agee, Ault and Emery, 1976. p. 77). Katz and Katz (1982) writes in Magazines for Libraries, that the New York Times and the Washington Post are the most recognized 
newspapers in America (p.705). The New York Times is generally considered the leading American daily and has a nationwide circulation. The Washington Post is well known for its "molding of a vigorous, intelligent and informative editorial page for Capital readers" (Agee, Ault and Emery, 1976. p.78). The Christian Science Monitor, with a circulation disproportionately smaller than the previous two, has a reputation for its objectivity and comprehensive coverage (Katz and Katz, 1982. P.704).

In 1978, five Japanese national dailies had a morning circulation of 23 million. Among these, three major newspapers, the Yomiuri Shimbun, the Asahi Shimbun and the Mainichi Shimbun control a 908 share of the total circulation (Yamamoto, 1980. p.33). The educational and informative contribution of Japanese newpapers in general and the large readership of these three newspapers throughout Japan in specific supports the choice of newspapers as good representatives of Japanese writing.

Therefore, the author chose the New York Times, the Christian Science Monitor and the Washington Post to represent the typically American style, and the Asahi Shimbun, the Yomiuri Shimbun and the Mainichi Shimbun to represent the typically Japanese style.

Editorials were chosen from these three major newspapers from each country. The first editorial appearing on the first day of the month between June 1981 and November 1981 were selected. The editorials were from the Yomiuri Shimbun and the Christian Science Monitor from their June and July issues, the Washington Post and the Asahi Shimbun from their August and September issues, and the New York Times and the Mainichi Shimbun from their October and November issues. Since the Christian Science Monitor is only published during the week, the following 
Monday issue was selected when the first of the month was either Saturday or Sunday.

The letters to the editor from two major newspapers from each country were chosen periodically, in this case every first letter which appeared in the issue on the first day of every month for ten months. Newspapers selected were the New York Times and the Christian Science Monitor for the American style sample and the Yomiuri Shimbun and the Asahi Shimbun for the Japanese style sample starting February 1981 to the November 1981 issue.

Thus altogether twenty letters and six editorials from each country were collected and rated in order to test the hypotheses.

\section{SELECTION OF RATERS AND SUBSAMPLES}

In order to establish whether or not the data coded by the author could provide a trustworthy basis for this research, nine outside raters were chosen to rate various subsamples of the editorials and letters.

Selection of the nine raters was based primarily on their formal education, since a more accurate analysis of variables to be rated could be expected from the person with above average education. The three Japanese raters have Masters of Art degrees in either Speech Communication or English. Among the six American raters, two hold Masters degrees in Liberal Arts, two have law degrees, one is an M.D. and one a B.A. The ages of the raters ranged from 27 to 39 for Americans and 31 to 33 for the Japanese. The raters were informed of the general research purpose but not of the specific hypotheses and were asked to rate subsamples according to the ten variables represented in 
the hypotheses.

The following subsamples of editorials and letters were assigned to raters to provide data for both the rater reliability test and for tesing the hypotheses (see Table I):

TABLE I

ORGANIZATION OF RATER AND SAMPLE POPULATION

\begin{tabular}{|c|c|c|c|c|c|}
\hline$\overline{\text { Rater }}$ & Sample & $\begin{array}{l}\text { \# of } \\
\text { edito rials }\end{array}$ & $\begin{array}{l}\text { \# of } \\
\text { let ters }\end{array}$ & \multicolumn{2}{|c|}{$\begin{array}{l}\text { Total \# of } \\
\text { coded samples }\end{array}$} \\
\hline 1 & U.S. + Japanese & 12 & 40 & 52 & \\
\hline 2 & \multirow{3}{*}{$\begin{array}{l}\text { a set of } 6 \text { distinct } \\
\text { U.S. samples }\end{array}$} & 2 & 4 & 6 & \multirow{3}{*}{18} \\
\hline 3 & & 2 & 4 & 6 & \\
\hline 4 & & 1 & 5 & 6 & \\
\hline 5 & \multirow{3}{*}{$\begin{array}{l}\text { a set of } 6 \text { distinct } \\
\text { Japanese samples }\end{array}$} & 2 & 4 & 6 & \multirow{3}{*}{18} \\
\hline 6 & & 2 & 4 & 6 & \\
\hline 7 & & 1 & 5 & 6 & \\
\hline 8 & \multirow{3}{*}{$\begin{array}{l}\text { a set of } 12 \\
\text { identical } \\
\text { bicultural samples }\end{array}$} & 4 & 8 & 12 & \multirow{3}{*}{36} \\
\hline 9 & & 4 & 8 & 12 & \\
\hline 10 & & 4 & 8 & 12 & \\
\hline
\end{tabular}

a.) Three American raters (rater 2, 3, 4) analyzed six distinct subsamples selected from American newspapers with respect to the ten variables of this study.

b.) Three Japanese raters (rater 5, 6, 7) examined six distinct subsamples selected from Japanese newspapers according to the same ten variables. 
c.) Three other American raters (rater 8, 9, 10) studied both six American subsamples and six Japanese subsamples which had been translated into English by this author. In this bicultural subsample, editorials and letters were arranged in random order and proper nouns were changed to mask the cultural source of the material, in order to eliminate any cultural bias.

\section{RELIABILITY TEST}

In order to record the different magnitude of characteristics of each variable, a set of ten seven-point bi-polar, verbally anchored rating scales was used by all raters, one per hypothesis, for each sample examined. The scores on the scale were then transformed into nominal data (see Figure 1). The instruction to the raters and a scoring sheet are shown in Appendix A. A summary of the coded samples per individual rater is shown in Appendix B.

LINEAR

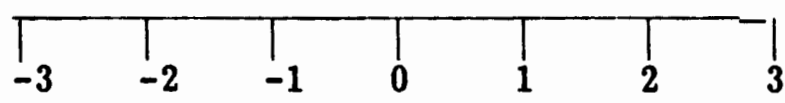

RANGE OF LINEARITY

RANGE OF NONLINEARITY

RANGE OF INDIFFERENCE
$-3 \leq$ RESULT $\leq-1$

$1 \leq$ RESULT $\leq 3$

NONLINEAR

$-1<$ RESULT< 1

\section{Figure 1. Rating scale example}

The first reliability test compared the author's (rater 1) rating of material to each individual rater in the two groups (raters 2-7) who rated either a purely Japanese or purely American sample of the same material. 
The agreement achieved between the author and raters was measured by percentage. The following Table II illustrates the agreement between rater 1 and raters 2 through 7 .

TABLE II

INTER-RATER AGREEMENT IN ANALYSIS OF SEPARATE SUBSAMPLES

\begin{tabular}{llllllll}
\hline RATER & 1,2 & 1,3 & 1,4 & 1,5 & 1,6 & 1,7 & Average \\
\hline $\begin{array}{l}\text { AGREE- } \\
\text { MENT }\end{array} 878$ & 788 & 928 & 888 & 838 & 828 & 858 \\
\hline
\end{tabular}

The agreement achieved between the raters (rater $1,8,9,10$ ) is shown in Table III. Rater 1 plus three other raters $(8,9,10)$ were given identical sets of bicultural subsamples, and the results were paired of with one another and tested in all possible rater combinations.

TABLE III

INTER-RATER AGREEMENT IN ANALYSIS OF TWELVE IDENTICAL BICULTURAL SUBSAMPLES

\begin{tabular}{llllllll}
\hline RATER 1,8 & 1,9 & 1,10 & 8,9 & 8,10 & 9,10 & Average \\
\hline $\begin{array}{l}\text { AGREE- } \\
\text { MENT }\end{array}$ 738 & 778 & 818 & 748 & 728 & 758 & 758 \\
\hline
\end{tabular}

Average inter-rater agreement from the first analysis was 858 (Table II), average inter-rater agreement from the second analysis (Table III) was 758 . 


\section{DATA ANALYSIS}

2

Chi-square ( $X$ ) estimates the probability of the occurrence under the $\mathrm{n}$ ull hypothesis based on the discrepancy between observed frequencies and expected frequencies in a set of categories. The ChiSquare value is yielded by:

$$
\mathrm{x}^{2}=\frac{\text { (Observed Frequencies - Expected Frequencies) }^{2}}{\text { Expected Frequencies }}
$$$$
\text { (Siegel, } 1956 \text { p. 104) }
$$

The Fisher Exact Probability test is used when either the sample number is smaller than 20 , or the sample size is between 20 and 40 with a smallest expected frequency of less than 5 , and if two independent samples fall into mutually exclusive categories. The calculation is based upon the marginal totals and observed frequencies in a $2 \times 2$ contingency table. The formula is:

$$
P=\frac{(A+B) !(C+D) !(A+D) !(B+D) !}{N ! A ! B ! C ! D !}
$$

Where $P$ is ... Probability

A-D are Observed Frequency in the Contingency

$\mathrm{N}$ is ... Sum of All Observed Frequencies

$$
\text { (Siegel, } 1956 \text { p.96) }
$$

The Chi-square test was used to determine the difference between the two sample groups in regard to all hypotheses except Hypothesis 5 (Results of Raters 2 through 7). The Fisher Exact Probability test was used there because the smallest expected frequency was less than five. 
rejection of the null statistical hypothesis. Since all hypotheses predict the direction of the difference between two independent groups, the region of rejection was one-tailed. In a Chi-square test, with df (degree of freedom $)=1, x^{2} \geq 2.71$ is required to reject the null statistical hypothesis at the $p=.05$ level.

In addition to the main analysis, each source was analyzed separately in light of the ten hypotheses. A Fisher Exact Probability test was applied to test for significant differences between editorials from both cultures. This supplementary test was based on rater 1 codes. The purpose of this test and the following Chi-square test was to determine if greater or less differences in style was attributable to either editorials or letters to the editor from each culture.

A Chi-square test was used to determine significant differences between let ters to the editor from both cultures. This supplementary test also was based on rater 1 codes.

Finally, a Fisher Exact Probability test was applied to test for significant differences between editorials and letters to the editor within each culture based on rater 1 codes. The purpose of this test was to see whether or not source material within one culture was different in style between editorials and letters to the editor. Table IV summarizes the three supplementary tests. 
TABLE IV

\author{
ORGANIZATION OF SUPPLEMENTARY TEST \\ FOR \\ CROSSCULTURE AND WITHIN-CULTURE DIFFERENCES
}

\begin{tabular}{|c|c|c|c|c|}
\hline Analysis & & & Test Applied & $\begin{array}{c}\text { Total \# } \\
\text { of Samples } \\
\end{array}$ \\
\hline Editorials & $\begin{array}{l}6 \mathrm{U} \cdot \mathrm{S} . \\
\text { editorials }\end{array}$ & $\begin{array}{l}6 \text { Japanese } \\
\text { editorials }\end{array}$ & Fisher & 12 \\
\hline \multirow[t]{2}{*}{$\begin{array}{l}\text { Letters to } \\
\text { the editor }\end{array}$} & $\begin{array}{l}20 \text { U.S. } \\
\text { letters }\end{array}$ & $\begin{array}{l}20 \text { Japanese } \\
\text { letters }\end{array}$ & Chi-square & 40 \\
\hline & $\begin{array}{l}6 \mathrm{U} . \mathrm{S} . \\
\text { editorials }\end{array}$ & $\begin{array}{l}20 \mathrm{U} \cdot \mathrm{S} . \\
\text { let ters }\end{array}$ & Fisher & 26 \\
\hline Within-culture & $\begin{array}{l}6 \text { Japanese } \\
\text { editorials }\end{array}$ & $\begin{array}{l}20 \text { Japanese } \\
\text { letters }\end{array}$ & Fisher & 26 \\
\hline
\end{tabular}




\section{CHAPTER IV \\ RESULTS AND DISCUSSION}

\section{RESULTS}

In this section the results for each hypothesis will be presented. The results are divided into two parts. Those 52 samples (12 editorials and 40 letters to the editor) rated by the author are called Results of Rater 1. Those 36 samples ( 10 editorials and 26 letters to the editor) judged by raters two to seven are called Results of Raters 2 through 7 . In a Chi-square test, $x^{2} \geq 2.71$ is required to reject the null statistical hypothesis at the $p=.05$. In a Fisher Exact Probability test, $p \leq .05$ is required to reject the null statistical hypothesis.

\section{Hypothesis 1}

The presentation of American arguments tends to be more linear whereas that of Japanese arguments tends to be more nonlinear.

The Chi-Square values obtained were 8.74 and 3.13 which are both sufficiently high to reject the first null statistical hypothesis. The observed frequencies for the "linearity" hypothesis and the statistical computations are presented in Table V and Table VI. (For the source of the underlying data (results of the coded samples) as basis of the following computation, refer to Appendix B.) 
TABLE V

LINEAR ITY OF PRESENTATION.

OBSERVED FREQUENCIES AND CHI-SQUARE COMPUTATION (RESULTS OF RATER 1)

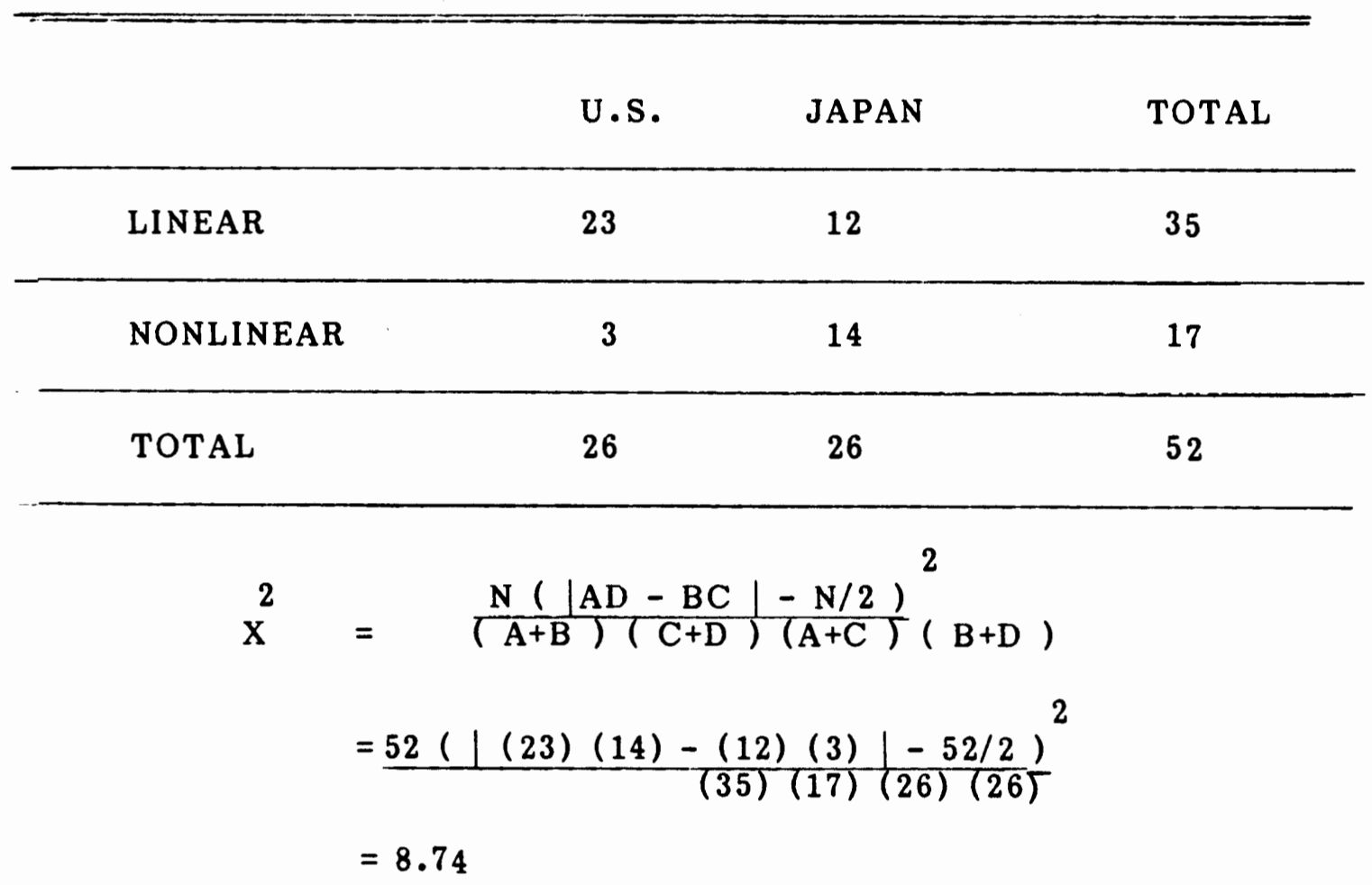


TABLE VI

LINEARITY OF PRESENTATION.

OBSERVED FREQUENCIES AND CHI-SQUARE COMPUTATION

(RESULTS OF RATERS 2 THROUGH 7)

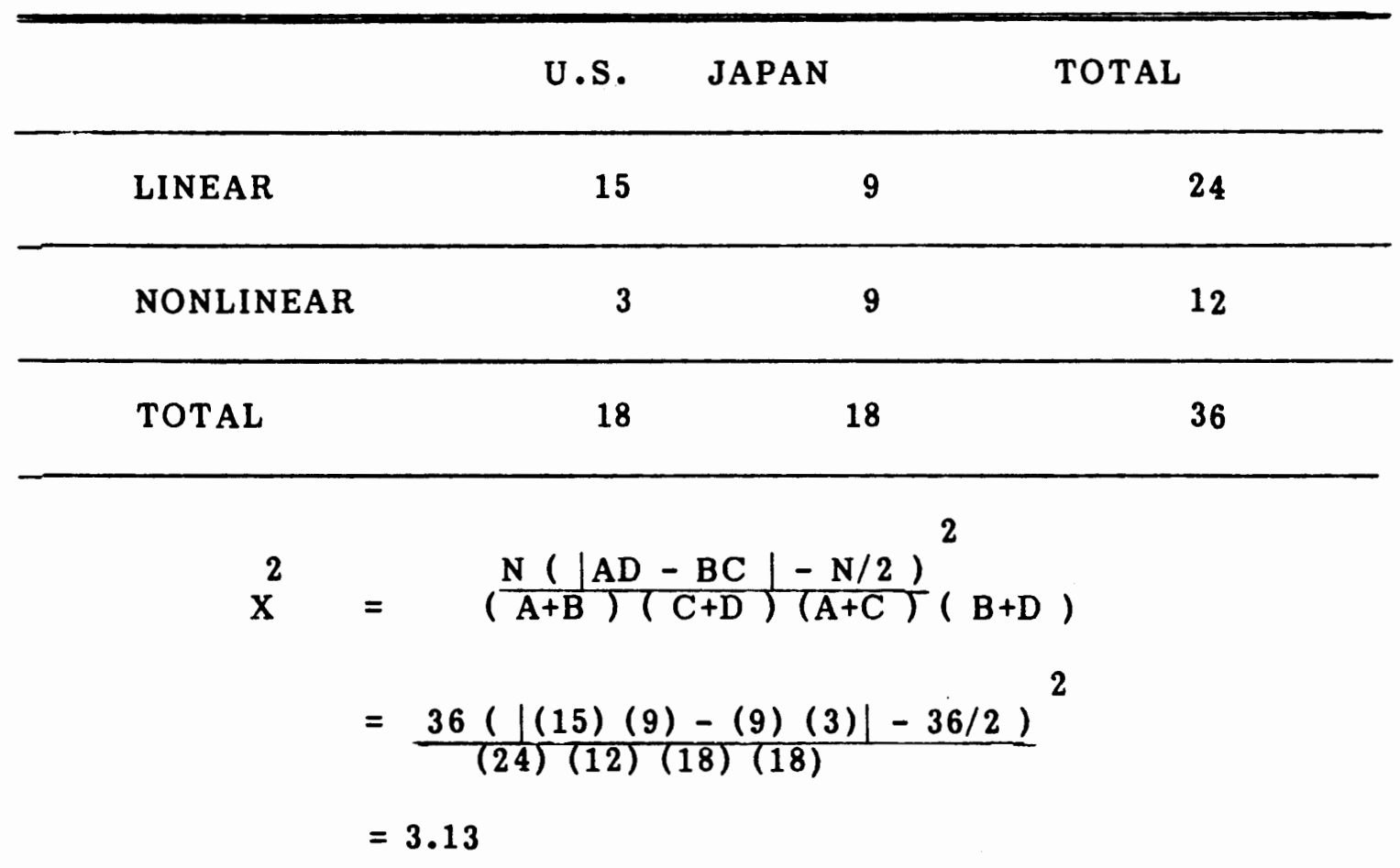

Hypothesis 2

American arguments tend to be more direct in their introduction of the subject matter than Japanese counterparts.

The Chi-Square values obtained were 6.56 and 3.13 which were both significant beyond the $p=.05$ level. The observed frequencies for the "directness" hypothesis and the statistical computations are presented in Table VII and Table VIII. 


\section{TABLE VII}

DIRECTNESS OF INTRODUCTION:

OBSERVED FREQUENCIES AND CHI-SQUARE COMPUTATION

( RESULTS OF RATER 1)

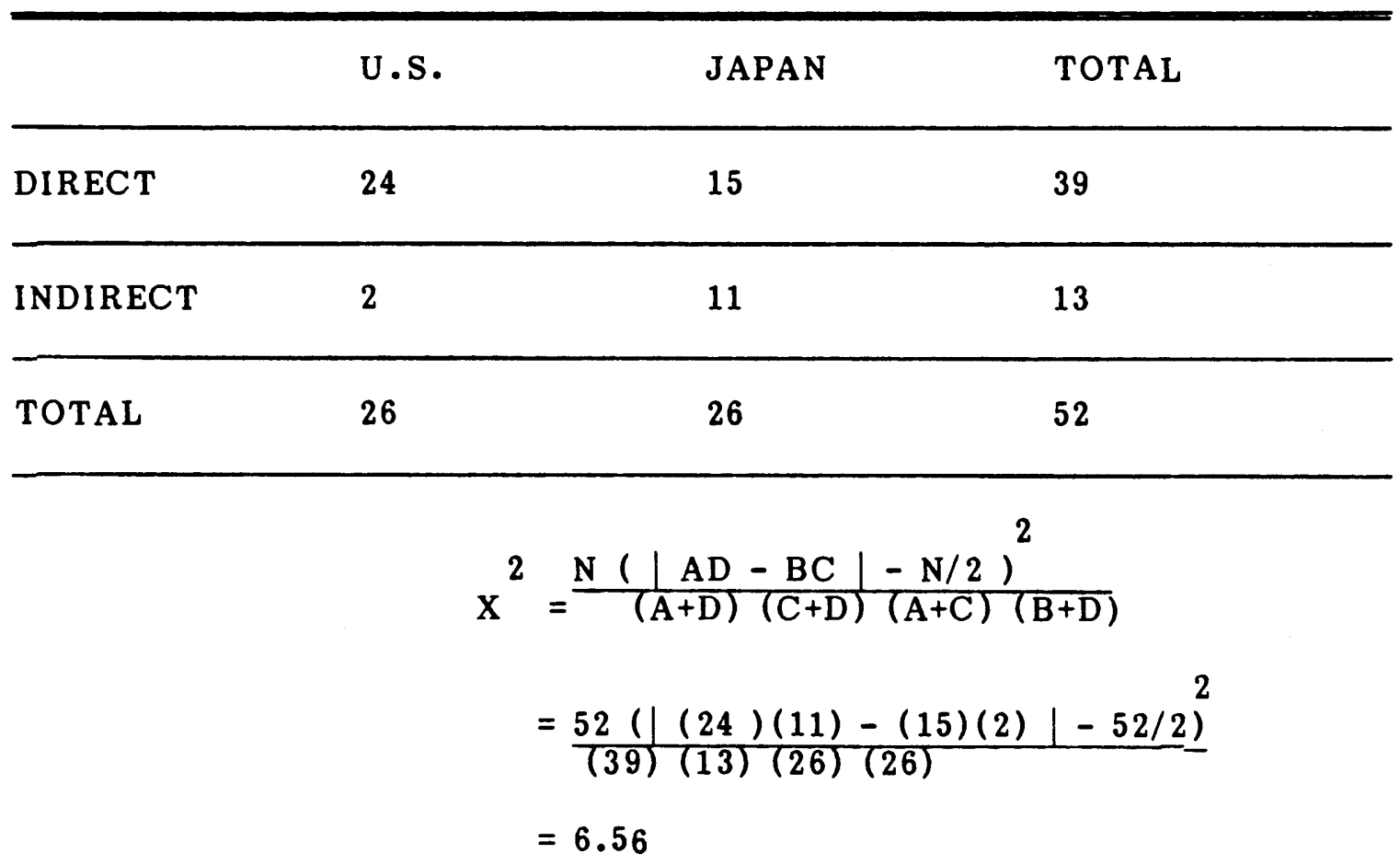


TABLE VIII

DIRECTNESS OF INTRODUCTION:

OBSERVED FREQUENCIES AND CHI-SQUARE COMPUTATION

( RESULTS OF RATERS 2 THROUGH 7 )

$\begin{array}{lll}\text { U.S. JAPAN TOTAL } & \end{array}$

\section{DIRECT}

15

9

24

INDIRECT

3

9

12

\section{TOTAL}

18

18

36

$$
\begin{aligned}
\mathbf{X}^{2} & =\frac{N(|A D-B C|-N / 2)^{2}}{(A+B)(C+D)(A+C)(B+D)} \\
& =\frac{36(|(15)(9)-(9)(3)|-36 / 2)}{(24)(12)(18)(18)}{ }^{2} \\
& =3.13
\end{aligned}
$$

Hypoth esis 3

In American argument there is less restatement of the same conclusion than in its Japanese counterpart.

The Chi-Square values obtained were 0.88 and 0 , not of sufficient magnitude to reject the null statistical hypothesis. The observed frequencies for "repetitiveness" hypothesis and the statistical computations are presented in Table IX and Table X. 
TABLE IX

REPETITIVENESS OF CONCLUSIONS:

OBSERVED FREQUENCIES AND CHI-SQUARE COMPUTATION

( RESULTS OF RATER 1)

\begin{tabular}{llll}
\hline & U.S. & JAPAN & TOTAL \\
\hline NONREPETITIVE & 17 & 21 & 38 \\
\hline REPETITIVE & 9 & 5 & 14 \\
\hline TOTAL & 26 & 26 & 52 \\
\hline
\end{tabular}

$$
\begin{aligned}
\mathrm{X}^{2} & =\frac{\mathrm{N}(|\mathrm{AD}-\mathrm{BC}|-\mathrm{N} / 2)^{2}}{(\mathrm{~A}+\mathrm{D})(\mathrm{C}+\mathrm{D})(\mathrm{A}+\mathrm{C})(\mathrm{B}+\mathrm{D})} \\
& =\frac{52(|(17)(5)-(21)(9)|-52 / 2)}{(38)(14)(26)(26)} \\
& =0.88
\end{aligned}
$$


TABLE $X$

REPETITIVENESS OF CONCLUSIONS:

OBSERVED FREQUENCIES AND CHI-SQUARE COMPUTATION

( RESULTS OF RATERS 2 THROUGH 7 )

U.S.

JAPAN

TOTAL

NONREPETITIVE 12

11

23

REPETITIVE

6

7

13

TOTAL

18

18

36

$$
\begin{aligned}
& 2 \\
& x^{2}=\frac{N(|A D-B C|-N / 2)}{(A+B)(C+D)(A+C)(B+D)} \\
& =\frac{36(|(12)(7)-(11)(6)|-36 / 2)^{2}}{(23)(13)(18)(18)} \\
& =0
\end{aligned}
$$

Hypothesis 4

American arguments tend to be more inductive or deductive than their Japanese counterparts.

There was a significant difference between both groups as to whether or not reasoning (either inductive or deductive) was used. After combining adjacent categories (inductive and deductive), a ChiSquare test was applied. The Chi-Square values obtained were 13.49 and 10.13, which fell in the region of rejection. The observed frequencies for the "inductive/deductive" hypothesis and statistical computation are presented in Table XI and Table XII. 
TABLE XI

TYPE OF REASONING:

OBSERVED FREQUENCIES AND CHI-SQUARE COMPUTATION

(RESULTS OF RATER 1)

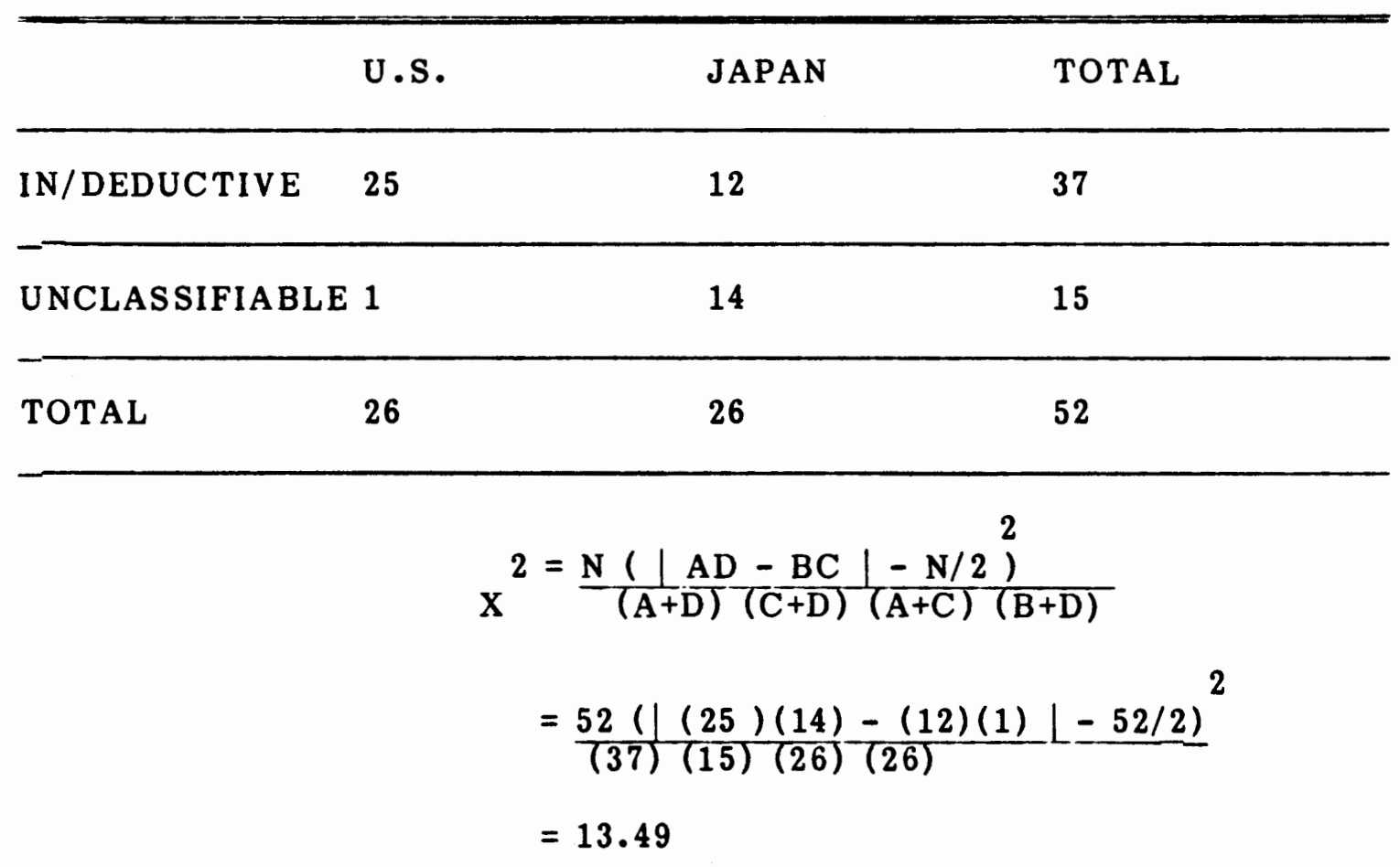


TABLE XII

TYPE OF REASONING:

OBSERVED FREQUENCIES AND CHI-SQUARE COMPUTATION

(RESULTS OF RATERS 2 THROUGH 7)

U.S.

JAPAN

TOTAL

IN/DEDUCTIVE 17

7

24

UNCLASSIFIABLE 1

11

12

TOTAL

18

18

36

$$
\begin{aligned}
X^{2} & =\frac{N(\mid A D-B C+-N / 2)^{2}}{(A+D)(C+D) \frac{-N}{(A+C)^{-}}(B+D)}- \\
& =\frac{36(|(17)(11)-(7)(1)|-36 / 2)^{2}}{(24)(12)(18)(18)} \\
& =10.13
\end{aligned}
$$

Hypoth esis 5

In American arguments, conclusions are more of ten explicitly stated than those in the Japanese arguments.

The Chi-square value obtained was 0 which was not statistically significant (Results of Rater 1). The Fisher Exact Probability test was used for Results of Raters 2 through 7 . The P yielded was 0.40 which did not show a difference between the two groups. Therefore the null statistical hypothesis was accepted. The observed frequencies for the "explicitness" hypothesis and statistical computations are presented in Table XIII and Table XIV. 
TABLE XIII

EXPLICITNESS OF CONCLUSIONS :

OBSERVED FREQUENCIES AND CHI-SQUARE COMPUTATION ( RESULTS OF RATER 1)

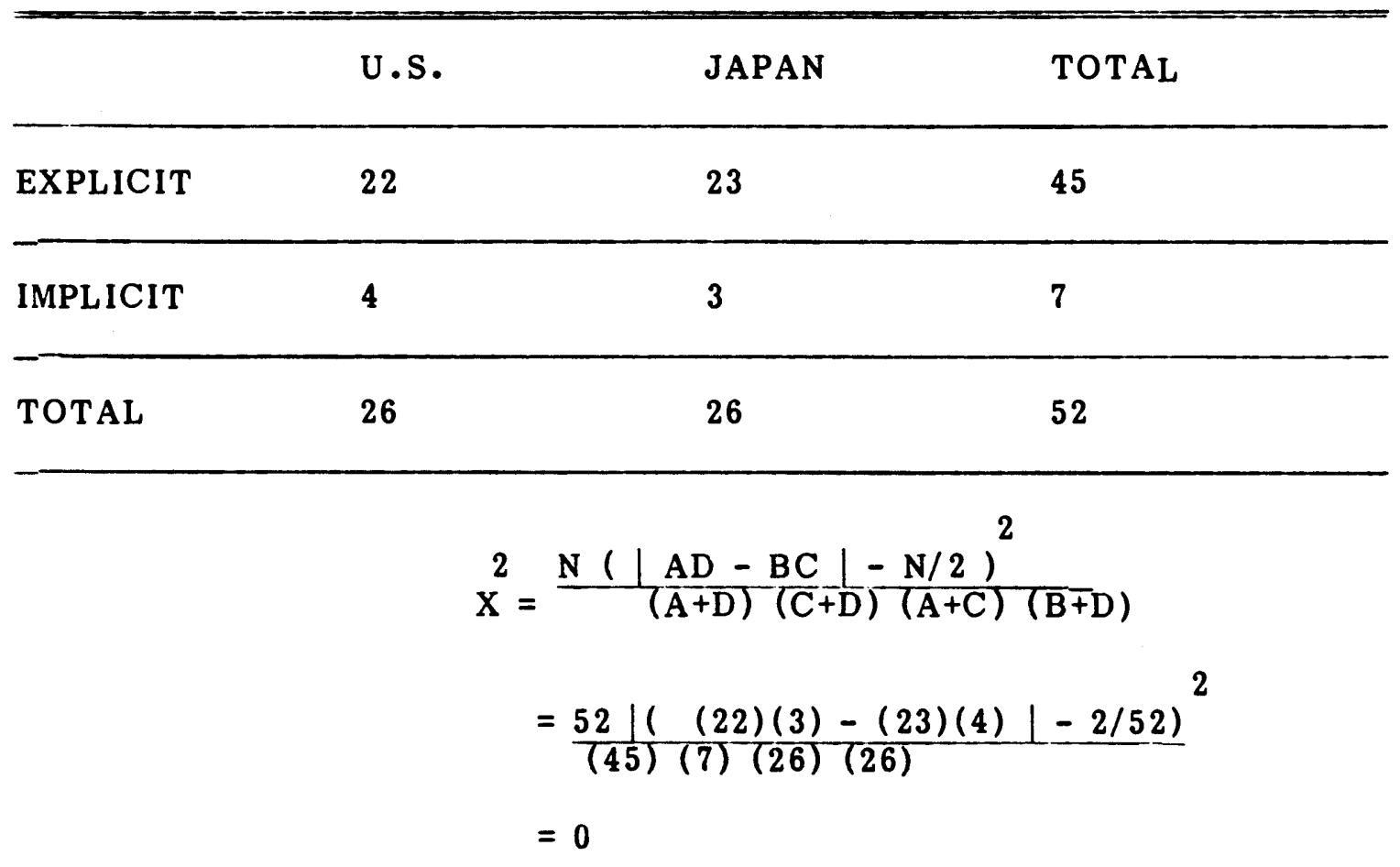


TABLE XIV

EXPLICITNESS OF CONCLUSIONS:

OBSERVED FREQUENCIES AND COMPUTAION OF FISHER EXACT

PROBABILITY

( RESULTS OF RATERS 2 THROUGH 7 )

\begin{tabular}{llll}
\hline & EXPLICIT & IMPLICIT & TOT AL \\
\hline U.S. & 16 & 2 & 18 \\
\hline JAPAN & 16 & 2 & 18 \\
\hline TOTAL & 32 & 4 & 36 \\
\hline
\end{tabular}

$$
\begin{aligned}
P & =\frac{(A+B) !(C+D) !(A+D) !(B+C) !}{N ! A ! B ! C ! D !} \\
& =\frac{18 ! 18 ! 32 ! 4 !}{36 ! 16 ! 2 ! 16 ! 2 !} \\
& =0.40
\end{aligned}
$$

Hypothesis 6

Statements in American arguments tend to be more concrete than abstract, whereas statements in Japanese arguments tend to be more abstract than concrete.

The Chi-Square values obtained were 1.22 and 0.12 , not of sufficient magnitude to reject the null statistical hypothesis. The observed frequencies for the "concreteness" hypothesis and the statistical computations are presented in Table XV and Table XVI. 
TABLE XV

CONCRETENESS OF STATEMENTS:

OBSERVED FREQUENCIES AND CHI-SQUARE COMPUTATION

( RESULTS OF RATER 1)

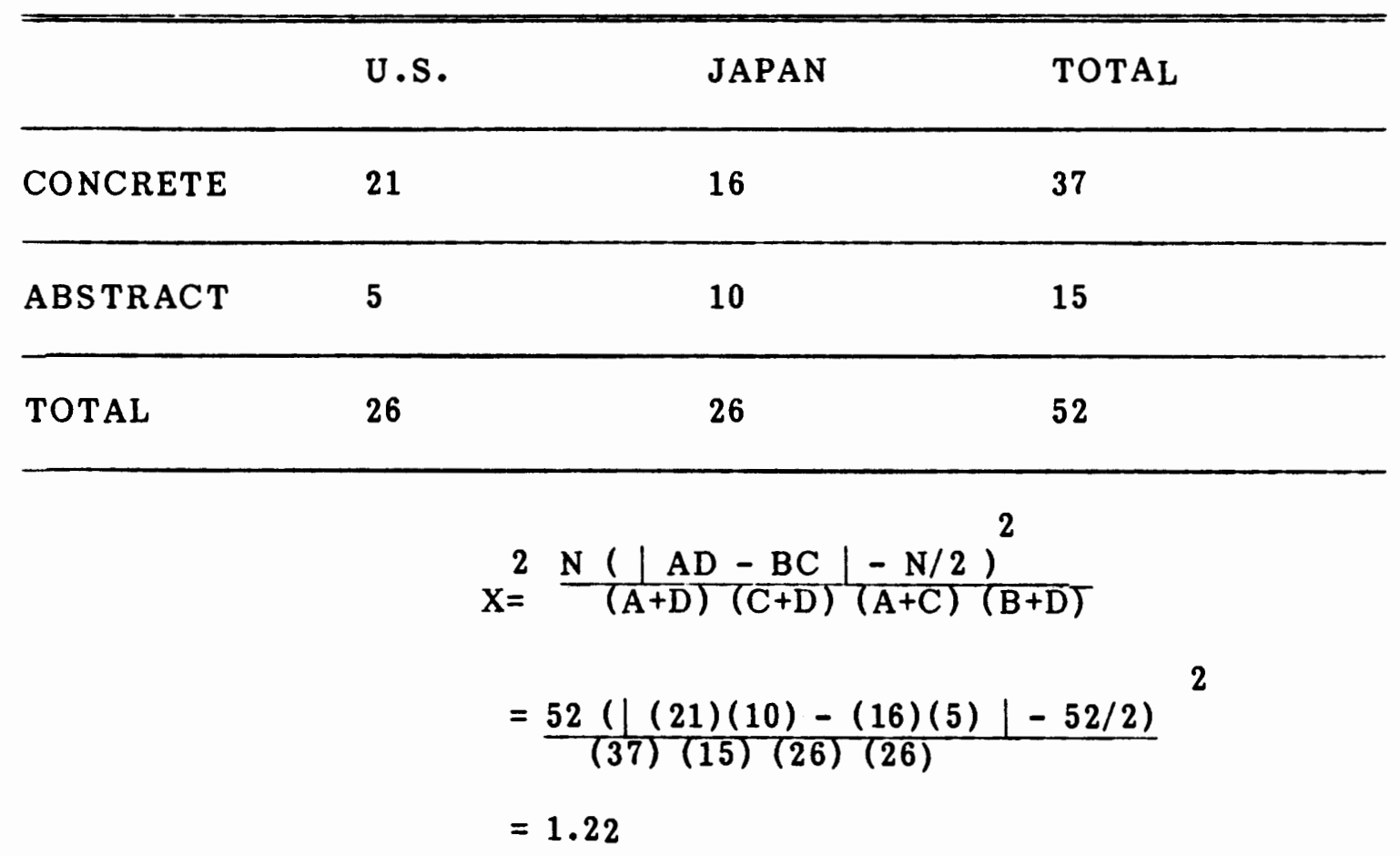


TABLE XVI

CONCRETENESS OF STATEMENTS:

OBSERVED FREQUENCIES AND CHI-SQUARE COMPUTATION

( RESULTS OF RATERS 2 THROUGH 7 )
U.S.
JAPAN
TOTAL

CONCRETE

12

10

22

ABSTRACT

6

8

14

TOTAL

18

18

36

$$
\begin{aligned}
& \mathrm{X}^{2}=\frac{\mathrm{N}(|\mathrm{AD}-\mathrm{BC}|-\mathrm{N} / 2)^{2}}{(\mathrm{~A}+\mathrm{B})(\mathrm{C}+\mathrm{D})(\mathrm{A}+\mathrm{C})(\mathrm{B}+\mathrm{D})} \\
& =\frac{36(|(12)(8)-(10)(6)|-36 / 2)}{(22)(14)(18)(18)} \\
& =0.12
\end{aligned}
$$

Hypothesis 7

Evidence in American arguments covers a wider perspective than that in Japanese arguments.

The Chi-Square value obtained was 4.99 which is statistically significant beyond the $p=.05$ level, however, the second analysis produced the result of 1.03 , failing to reject the null statistical hypothesis. The observed frequencies for the "width" hypothesis and the computations are presented in Table XVII and Table XVIII. 
TABLE XVII

WIDTH OF PERS PECTIVE:

OBSERVED FREQUENCIES AND CHI-SQUARE COMPUTATION ( RESULTS OF RATER 1)

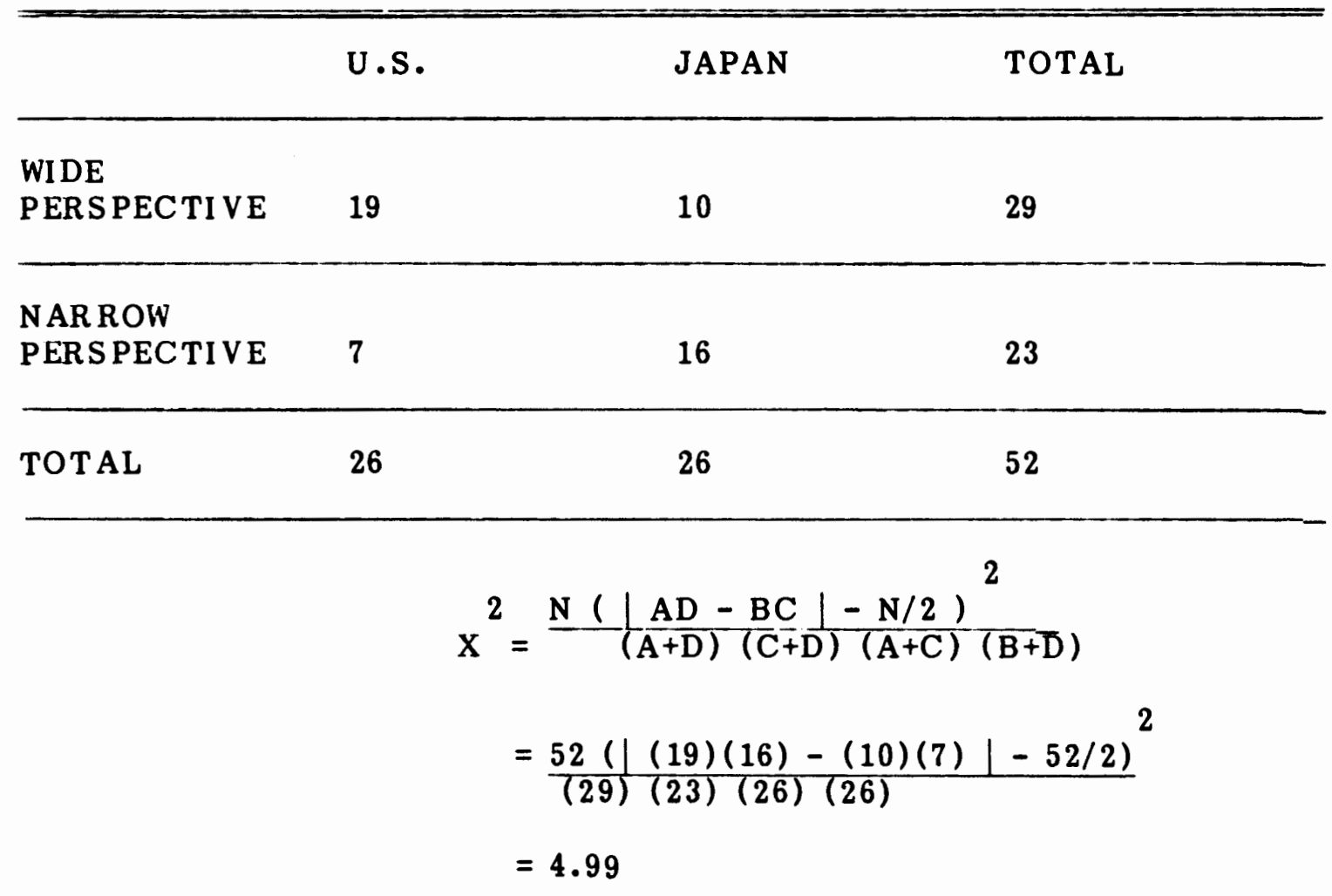




\begin{tabular}{llll}
\hline & U.S. & JAPAN & TOTAL \\
\hline $\begin{array}{l}\text { WIDE } \\
\text { PERS PECTIVE }\end{array}$ & 12 & 8 & 20 \\
\hline $\begin{array}{l}\text { NAR ROW } \\
\text { PERS PECTIVE }\end{array}$ & 6 & 10 & 16 \\
\hline TOT AL & 18 & 18 & 36 \\
\hline
\end{tabular}

$$
\begin{aligned}
x^{2} & =\frac{N(|A D-B C|-N / 2)^{2}}{(A+B)(C+D)(A+C)(B+D)} \\
& =\frac{36(|(12)(10)-(8)(6)|-36 / 2)}{(20)(16)(18)(18)} \\
& =1.03
\end{aligned}
$$

Hypothesis 8

Definite and strengthening qualifiers appear more frequently in statements of American arguments than in their Japanese counterparts, where conditional and weakening qualifiers are found more frequently.

The Chi-Square values obtained were 13.11 and 10.13 which are statistically significant beyond the $p=.05$ level. The observed frequencies for the "definiteness" hypothesis and the statistical computations are presented in Table XIX and Table XX. 
TABLE XIX

DEFINIT ENESS OF QUALIFIERS:

OBSERVED FREQUENCIES AND CHI-SQUARE COMPUTATION ( RESULTS OF RATER 1 )

U.S.

26

DEFINITE

CONDITIONAL

TOTAL

0

26
JAPAN

14

12

12

TOTAL

40

52

$$
\begin{aligned}
X^{2} & =\frac{N(|A D-B C|-N / 2)^{2}}{(A+D)(C+D)(A+C)(B+D)} \\
& =\frac{52(\mid(26)(12)-(14)(0) L-52 / 2)^{2}}{(40)(12)(26)(26)} \\
& =13.11
\end{aligned}
$$


TABLE XX

DEFINITENESS OF QUALIFIERS :

OBSERVED FREQUENCIES AND CHI-SQUARE COMPUTATION

( RESULTS OF RATERS 2 THROUGH 7 )
U.S.
JAPAN
TOTAL

\section{DEFINITE}

17

7

24

CONDITIONAL 1

11

12

\section{TOTAL}

18

18

36

$$
\begin{aligned}
& \mathrm{X}^{2}=\frac{\mathrm{N}(\mid \mathrm{AD}-\mathrm{BC}}{(\mathrm{A}+\mathrm{B})(\mathrm{C}+\mathrm{D})(-\mathrm{N} / 2)^{2}}
\end{aligned}
$$

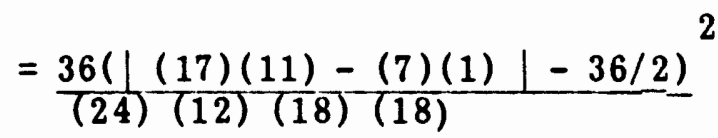

$$
\begin{aligned}
& =10.13
\end{aligned}
$$

\section{Hypothesis 9}

Evidence in American arguments tends to be more objective than that of Japanese counterparts, whereas evidence in Japanese arguments tends to be more subjective than in American counterparts.

The Chi-Square value obtained was 3.86 which is sufficiently high to reject the null statistical hypothesis(Results of rater 1), however, the Chi-square value obtained by Results of Raters 2 through 7 was 0.12 , not of sufficient magnitude to reject the null statistical hypothesis. The observed frequencies for the "objectivity" hypothesis and statistical computations are presented in Table XXI and Table XXII. 


\section{TABLE XXI}

OBJECTIVITY OF EVIDENCE:

OBSERVED FREQUENCIES AND CHI-SQUARE COMPUTATION ( RESULTS OF RATER 1 )

\begin{tabular}{|c|c|c|c|}
\hline & U.S. & JAPAN & TOTAL \\
\hline OBJECTIVE & 19 & 11 & 30 \\
\hline SUBJECTIVE & 7 & 15 & 22 \\
\hline \multirow[t]{3}{*}{ TOTAL } & 26 & 26 & 52 \\
\hline & \multicolumn{3}{|c|}{$\frac{N(|A D-B C|-N / 2)^{2}}{(A+D)(C+D)(A+C)(B+D)}$} \\
\hline & \multicolumn{3}{|c|}{$=\frac{52(|(19)(15)-(11)(7)|-52 / 2)}{(30)(22)(26)(26)}$} \\
\hline
\end{tabular}


TABLE XXII

OBJECTIVITY OF EVIDENCE:

OBSERVED FREQUENCIES AND CHI-SQUARE COMPUTAION

( RESULTS OF RATERS 2 THROUGH 7 )

U.S.

7

11 18

11

18

JAPAN

7

11 18

11
TOTAL

SUBJECTIVE

22

TOTAL

$$
\begin{aligned}
\mathrm{X}^{2} & =\frac{\mathrm{N}(|\mathrm{AD}-\mathrm{BC}|-\mathrm{N} / 2)^{2}}{(\mathrm{~A}+\mathrm{B})(\mathrm{C}+\mathrm{D})(\mathrm{A}+\mathrm{C})(\mathrm{B}+\mathrm{D})} \\
& =\frac{36(\mid)(7)(11)-(7)(11) \mid-36 / 2)^{2}}{(14)(22)(18)(18)} \\
& =0.12
\end{aligned}
$$

Hypothesis 10

American arguments tend to include more analytical statements, whereas Japanese arguments have more emotional statements.

The Chi-Square values obtained were 2.12 and 0.11 respectively, not of sufficient magnitude to reject the null statistical hypothesis. The observed frequencies for the "analyticity" hypothesis and the statistical computations are presented in Table XXIII and Table XXIV, followed by the summarized tabulation of the ten hypotheses tested in Table XXV. 
T ABLE XXIII

ANALYTICITY OF STATEMENTS:

OBSERVED FREQUENCIES AND CHI-SQUARE COMPUTATION

( RESULTS OF RATER 1)

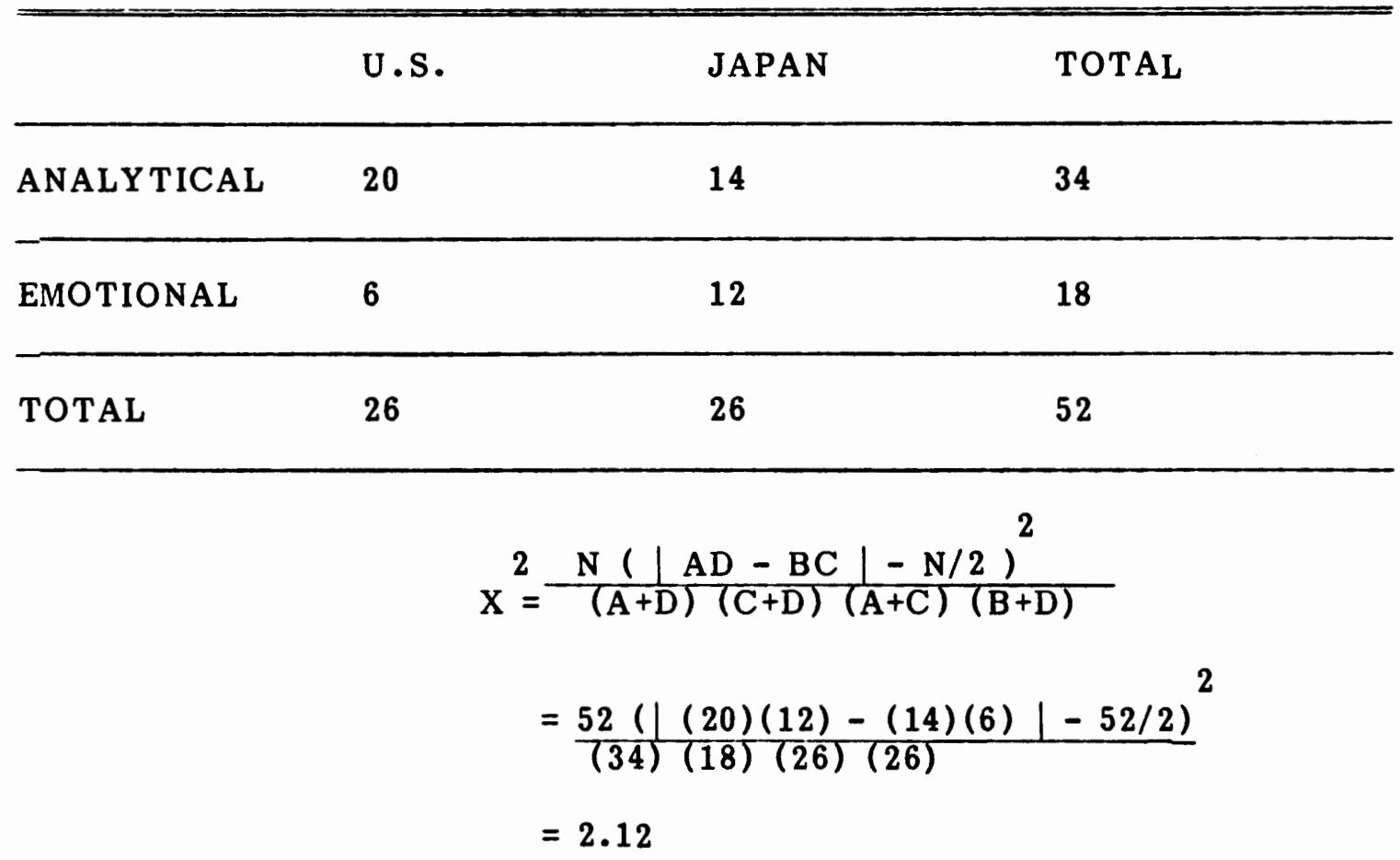


TABLE XXIV

ANALYTICITY OF STATEMENTS:

OBSERVED FREQUENCIES AND CHI-SQUARE COMPUTATION

( RESULTS OF RATERS 2 THROUGH 7 )

\begin{tabular}{llll}
\hline \hline & U.S. & JAPAN & TOTAL \\
\hline ANALYTICAL & 10 & 8 & 18 \\
\hline EMOTIONAL & 8 & 10 & 18 \\
\hline TOTAL & 18 & 18 & 36 \\
\hline
\end{tabular}

$$
\begin{aligned}
X^{2} & =\frac{N(|A D-B C|-N / 2)^{2}}{(A+B)(C+D)(A+C)}(\overline{B+D}) \\
& =\frac{36(|(10)(10)-(8)(8)|-36 / 2)}{(18)(18)(18)(18)} \\
& =0.11
\end{aligned}
$$


TABLE XXV

SUMMARIZED TABULATION OF THE TEN HYPOTHESES TESTED

\begin{tabular}{lrrrr}
\hline Hy pothesis & \multicolumn{1}{c}{$\mathrm{X}^{2}$} & $\mathrm{~S}$ & \multicolumn{1}{c}{$\mathrm{X}^{2}$} & $\mathrm{~S}$ \\
\hline 1. Linearity & 8.74 & $\mathrm{~S}$ & 3.13 & $\mathrm{~S}$ \\
2. Directness & 6.56 & $\mathrm{~S}$ & 3.13 & $\mathrm{~S}$ \\
3. Repetitiveness & 0.88 & & 0 & \\
4. Reasoning & 13.49 & $\mathrm{~S}$ & 10.13 & $\mathrm{~S}$ \\
5. Explicitness & 0 & & $\mathrm{P}=0.40$ & \\
6. Concreteness & 1.22 & & 0.12 & \\
7. Width & 4.99 & $\mathrm{~S}$ & 1.03 & $\mathrm{~S}$ \\
8. Qualifiers & 13.11 & $\mathrm{~S}$ & 10.13 & \\
9. Objectivity & 3.86 & $\mathrm{~S}$ & 0.12 & \\
10. Analyticity & 2.12 & & 0.11 & \\
\hline
\end{tabular}

Results of

Rater 1
Results of

Raters 2 through 7

S: SIGNIFICANT

Editorials Analysis

Since the size of the sample was small $(N=12)$, editorials from both groups were compared using the Fisher Exact Probability test. Table XXVI illustrates the results of the Fisher Exact Probability test between American editorials and Japanese editorials in terms of the ten variables. 
TABLE XXVI

EDITORIALS ANALYSIS

\begin{tabular}{ll}
\hline Hypothesis & $P$ \\
\hline 1 & 0.23 \\
2 & 0.09 \\
3 & 0.54 \\
4 & 0.09 \\
5 & 1 \\
6 & 0.55 \\
7 & 1 \\
8 & 0.23 \\
9 & 0.5 \\
10 & 0.5 \\
\hline
\end{tabular}

There were no significant differences in any categories between American newspaper editorials and Japanese editorials.

Letters to the Editor Analysis

The Chi-Square test was used (except \#5, Fisher) to determine whether there were significant differences between American letters and Japanese let ters to the editor. Table XXVII presents the results of the analysis between American and Japanese letters to the editor. 
TABLE XXVII

LETTERS ANALYSIS

\begin{tabular}{ccc}
\hline Hypothesis & \multicolumn{2}{l}{ X } \\
\hline 1 & 8.64 & $\mathrm{~S}$ \\
2 & 3.33 & $\mathrm{~S}$ \\
3 & 1.07 & \\
4 & 5.26 & $\mathrm{~S}$ \\
5 & 0.30 & $($ Fisher $)$ \\
6 & 1.82 & \\
7 & 6.55 & $\mathrm{~S}$ \\
8 & 10.8 & $\mathrm{~S}$ \\
9 & 6.42 & $\mathrm{~S}$ \\
10 & 3.68 & $\mathrm{~S}$ \\
\hline
\end{tabular}

S: SIGNIFICANT

Significant differences were observed in hypotheses $1,2,4,7,8,9$ and 10.

Within-culture Analysis

The significance of difference between editorials and letters to the editor within a culture was computed using the Fisher Exact Probability test based on codes of rater 1 . Table XXVIII shows the value of $P$ obtained within either American or Japanese newspapers in terms of the ten variables. 


\section{TABLE XXVIII}

\section{WITHIN-CULTURE ANALYSIS}

EDITORIALS VS. LETTERS

\begin{tabular}{cll} 
Hypoth esis & U.S. & JAPAN \\
\hline 1 & $\mathrm{P}=0.44$ & $\mathrm{P}=0.20$ \\
2 & $\mathrm{P}=0.58$ & $\mathrm{P}=0.33$ \\
3 & $\mathrm{P}=0.24$ & $\mathrm{P}=0.44$ \\
4 & $\mathrm{P}=0.77$ & $\mathrm{P}=0.35$ \\
5 & $\mathrm{P}=0.17$ & $\mathrm{P}=0.44$ \\
6 & $\mathrm{P}=0.44$ & $\mathrm{P}=0.18$ \\
7 & $\mathrm{P}=0.12$ & $\mathrm{P}=0.0005 \mathrm{~S}$ \\
8 & $\mathrm{P}=1$ & $\mathrm{P}=0.29$ \\
9 & $\mathrm{P}=0.35$ & $\mathrm{P}=0.002 \mathrm{~S}$ \\
10 & $\mathrm{P}=0.40$ & $\mathrm{P}=0.013 \mathrm{~S}$ \\
\hline
\end{tabular}

\section{S: SIGNIFICANT}

No significant differences were observed between editorials and letters to the editor sampled from American newspapers. The significant differences were observed in hypotheses 7,9 and 10 between editorials and letters to the editor from Japanese newspapers. 
DISCUSSION AND IMPLICATION FOR INTERCULTURAL COMMUNICATION

Hypothesis 1 (linear/nonlinear)

Results supported the research hypothesis that the presentation of American arguments tend to be more linear than that of the Japanese, whereas Japanese arguments tend to be more nonlinear than that of the Americans. The result was predominantly determined by the difference between American letters to the editor and Japanese let ters to the editor, since comparison of the two sets of editorials showed no significant difference. The views of Kunihiro, Kaplan et al., showing linearity of style among Americans and nonlinearity among Japanese, were supported by the result of the study.

Implication for Intercultural Communication. People with a predominantly linear orientation would perceive a nonlinear presentation as being out of focus, lacking in organization and having insufficient cohesion, and thus may label others with nonlinear orientation to be unintelligible communicators. The tendency toward a presentation interspersed with unrelated and of ten irrelevant elements might prevent Japanese from writing well-organized scholarly papers in the academic world or discussing foreign policies in an intercultural setting. The lack of developing strong internal continuity through the use of logic and reasoning in their attempt to communicate could be compared by an American to the multiple ambiguity of a Rorschach blot. Such a presentation may well be received as a message by the Japanese who are aware of context and have interpretatory strategies for nonlinear communication, but for the uninitiated Westerner, it may contain just more unanswered questions in an abundance of vagueness.

From a nonlinear point of view, the step-by-step approach of 
Americans seems either unnecessarily elaborate or too condescendingly direct and too manipulative to allow for the audience's insight or imagination.

Hearing an unfamiliar noise outside, a Japanese might typically comment "water, pavement, a street". Provided with these message fragments, a Japanese listener would most likely understand what is happening. On the same occasion, an American might remark, "Where is this noise coming from? Ah, there is a street sweeper in front of the building". This would illustrate the Westerner's tendency to correlate the sensory input into a factually acceptable and reasonably logical conclusion.

Thus it is obvious that each group, when interacting with each other, violates the other's expectation, which may later cause a mutually erroneous value judgment toward the other group as a whole.

\section{Hypothesis $2 \quad$ (direct/indirect)}

An American preference for the more direct introduction of the subject was observed as compared to that of the Japanese, based on the principal difference in the letters to the editor sampled from both groups. The present study supported Kaplan, Kobayashi et al. who discussed the United States' immediacy and the indirectness of the Japanese.

Implication for Intercultural Communication. A straight forward approach may well intimidate a person whose culturally conditioned preference for the introduction of a subject is one of indirectness. Those in the habit of coming straight to the point of ten become frustrated with a long introduction, which in their eyes does not necessarily relate or contribute to the subject. $\Gamma$ In a business situation, it is important to the 
Japanese to spend some time to become acquainted with each other before both parties start discussing business. This indirect and deliberate approach of the Japanese is of ten interpreted as a lack of interest in the business at hand by the American side. From the Japanese point of view, the direct approach seems too functional, impersonal and crass since they prefer building a limited but firm personal relationship with the other side prior to conducting business.

Thus the universal unconscious preconception about the similarity among people is followed by a judgment of the other from the point of one's own communicative behavior pattern and brings frequent unnecessary conflicts, whereas a sensitivity to conceive an alternate behavior pattern would allow for more constructive and nonjudgmental communication.

Hypothesis 3 (non repetitive/repetitive)

The present study did not show a Japanese tendency to use repetitive conclusions. Most of the Americans and the Japanese generally stated their conclusions once. The Americans repeated conclusions predominantly as a part of deductive reasoning whereas Japanese repetitive conclusions were simply restatements without reference to material presented before or thereafter. In conjunction with a similarity observed in terms of explicitness of conclusions, a correlating similarity in the number of conclusions might result from the degree of formality of the written discourse. A future analysis will be necessary to determine the significance of the differences between written and oral discourse, if any. 
Hypothesis 4 (inductive/deductive)

There was a significant difference observed in terms of whether inductive, deductive reasoning or neither was used. Americans predominantly use either inductive or deductive reasoning, whereas Japanese do not apply either of those forms of reasoning as frequently as Americans. Closely correlated to a Japanese tendency toward nonlinearity, results revealed a culturally conditioned lack of propensity for deductive abstraction by the Japanese. They neither proceed from the specific detail to a general assertion, nor from a generally accepted conclusion to a specific point that would necessarily be considered as an essential part of developing the principal argument. The observation by Kobayashi, Kaplan et al., that a greater emphasis is placed on inductive/deductive reasoning by Americans and a lesser emphasis by Japanese, is supported by this study.

Implication for Intercultural Communication. There is substantial factual support to the argument that logical development and consistency are essential ingredients of the American pattern of communication. Consequently, people in the United States observe foreign af fairs and attempt to predict the final outcome in adherence to and trust of their line of reasoning, and thus of ten feel disappointment and frustration from the apparently illogical and thus unpredictable consequences of political events in foreign countries. Americans perceive an outcome which is not logically comprehensible as illogical, irrational and undesirable. Taking former Japanese Prime Minister Tanaka's scandal as an illustrating example, it would be very difficult to understand by most Americans who accused Nixon of his illegal conduct that Mr. Tanaka still serves in public of fice today. 
The Japanese, whose psychological dependency upon one another is based on the exchange of honne, the emotional self, which is more important than tatemae, the logical self, might comment on that same scandal that this would seem a mere contradiction to the logic of ethics to them, however, that there is a more important factor beyond logic. For the Japanese, logic is not the principal means but only one of the means of determining right and wrong. Applying such logic in all aspects of life seems just too simplistic and idealistic, and although they might experience a feeling of moral outrage, they may feel collectively powerless to translate this into action. Thus a feeling of deepseated reluctance to actively change the status quo of their socio-economic condition, from their personal to their public life, contributes further to the prevailing pragmatic view of ap parent conformism and political inertia. This attitude makes Tanaka's continuation as a powerful and highly influential public servant not unusual for the Japanese.

Thus, both groups, expecting the other to be similar to themselves, fail to understand each other as they are.

Hypothesis 5 (explicit/implicit)

The present research did not support the difference between explicit and implicit nature of the conclusion which is discussed by Mushakoji, Hall et al. Instead, both samples showed a predominant explicitness. This might result from the fact that the present study only dealt with a formal discourse aimed at a mass audience.

Hypothesis $6 \quad$ (concrete/abstract)

Both an American and Japanese preference for concrete statements 
was observed. It appears that letters to the editor, in particular, were generally written about the writer's immediate experiences, therefore, both groups favored specific and descriptive statements over broad and theoretical statements.

Hypothesis 7 (wide/narrow)

There was a significant difference observed in terms of the total width of perspective. American writers predominantly use more evidence to support their conclusions than the Japanese. The most significant difference between the two cultures was shown by the letters to the editor. Results support the observation of Bernstein, Hall et al.

Implication for Intercultural Communication. Parallel to the findings of Hypothesis 9 (objectivity of evidence), this result strongly reflects the American preference for building a firm basis to support their conclusions, whereas the Japanese appear to be less eager to provide as much evidence. The apparently ambiguous communication by the Japanese would not provide sufficient evidence to the Americans in order to reach them with the desired degree of persuasion, as they lack that preassumed base of evidence.

Hypothesis 8 (definite/conditional)

A very significant difference was found in terms of the use of qualifiers. Americans predominantly use definite qualifiers whereas the Japanese favor more conditional qualifiers. Results supported the observation by Suzuki, Condon et al., that Japanese exhibit a preference for conditional (ambiguous) communication and the Americans a preference for definite (specific) communication. 
Implication for Intercultural Communication. The repeated use of

conditional qualifiers by the Japanese may be perceived as a lack of confidence and decisiveness by Americans who prefer clear communication. Definite statements by the Americans give the Japanese the impression of unwarranted and excessive confidence, oversimplification and insistence. American businessmen therefore may misinterpret the relative degree of commitment by the Japanese using an apparently ambiguous and interpretative style. The interaction between people of both cultures has commonly resulted in the misinterpretation of each other's relative position, thoroughness and degree of commitment and will continue to do so unless each side learns to understand and accept the other's dissimilarity without being unnecessarily judgmental.

\section{Hypothesis 9 (objective/subjective)}

A significant difference was found in the use of either objective evidence or subjective evidence. Results indicate that although subjective evidence is predominantly favored by the Japanese, editorial staff use more objective evidence than other Japanese. The present study supported Matsumoto's discussion, i.e. a tendency toward objectivity among Americans and subjectivity among the Japanese.

Implication for Intercultural Communication. Americans, who rely heavily on objective evidence, give Japanese the impression of being impersonal, functional and coldly detached. This is caused by Americans not sharing similar values among themselves as most Japanese do, and who therefore attempt to use the elaborated code.

Subjective evidence of ten used by the Japanese seems to be effective only among people who share similar background and feelings. 
The attitude of Japanese, dependent on their similarity of background and their need to be passively understood, would puzzle Americans who rely predominantly on objectivity in conjunction with logic. The mutual expectation of a crosscultural similarity results in one of the major stumbling blocks to the clear understanding of one another.

Hypothesis 10 (analytical/emotional)

Although the letters to the editor sampled from the two groups showed a significant difference in terms of American writers using more analytical statements than the Japanese, no overall significant difference was found in terms of the statement analysis.

An additional point of interest was to see whether there was significant variance between the letters to the editor and editorials within each country. A short statistical analysis of these questions showed no significant difference in style of letters to the editor and editorials in the United states, but a significant difference in style between letters to the editor and editorials in Japan. Editorials from both countries showed no significant difference when tested against one another, whereas letters from both countries showed significant differences in hypotheses $1,2,4$, $7,8,9$, and 10 . Therefore the differences observed in this study are due to the different styles used by letter writers of the two culture. The similarity of style between Japanese and American editorials indicated a shift of the Japanese editorial writer toward the typically Western format to an extent as to make the typical distinctions of style insignificant. By inference it may be assumed that Western influence of style and rhetoric is most profound on Japanese editorial staff. Furthermore, since letters 
to the editor are routinely edited by newspaper staff for brevity and clarity, it is reasonable to expect that the original letters contained more elements of ambiguity, repetitiveness (Japan) and elaboration of facts (United States).

\section{LIMIT ATION OF THE STUDY}

The limitation of this study should be noted and its findings should be construed in light of its limitations.

First, the present study dealt only with aspects of arguments among written discourses which were deliberately prepared by their authors as addressed to a mass audience. While it is possible that spoken arg uments may employ different strategies from that of written discourses, the theorists (Samovar \& Porter, 1982. p. 32) mentioned earlier support the essential connection of oral and written style as indicative of a culturally influenced organizational pattern. Nevertheless, another study focusing only on oral argumentative style would be useful in completing a comprehensive view of the differences noted in this study.

Second, sampling newspapers with similarity of function and readership in both countries was difficult. Since the three newspapers used as Japanese sample sources make up 908 of the total circulation, it was hard to find equivalent newspapers in the United States with a comparable universality of readership and similar cross section of social strata, since newspapers in America are virtually all regional in content and character and furthermore are appealing to a narrower social segment of their potential readers.

Third, the author, although a native Japanese, had difficulty in placing finite conclusions in some of the Japanese articles sampled. It 
seemed that, like haiku poetry, there were several interpretations of the article possible depending upon the reader's view of the writer's apparent conclusion.

\section{SUGGESTION FOR FUTURE RESEARCH}

There are several issues in need of clarification in future studies regarding the styles of arguments. 1) An analysis of the distinction between the formal and informal written discourse and the formal and informal oral one, impromptu speech in particular. 2) Advertising script would be a useful medium to determine the use of variables discussed in this study, since it is an abbreviated, quintessential method to get across a message, a fact, a request or demand or a feeling of need and desire, couched in direct language or the oblique, the hard sell or the innuendo. A study on the use of $\mathrm{TV}$ commercials as a representative reflection of the style of communication shows a recent trend to use additional communicative media to study and understand the effect and effectiveness of communication within and between both cultures (Huruse, 1978). 3) Further suggested substudies which may add to a deeper understanding of the subject may include, but not be limited to: a) gender-oriented differences; b) stratification along educational lines; and c) distinction in approach by age class, if any. 


\section{CHAPTER V}

\section{PERSONAL EXPER IENCES}

The author, a native Japanese, has attempted to use the American style of argument in the process of writing her thesis. The entire manner of writing this thesis was the method of putting the different argumentative strategies under discussion into practice.

Initially, the most difficult work was to break down the topic into subsections and to develop an analytical outline which sketches the content, reasoning and entire structure of the thesis. After refining the outline, the skeleton of the entire paper was complete and each element logically interrelated with the others. Writing a coherent outline by Western standards is the most essential part of writing a thesis. However, the author had an inherent tendency to write the content without an awareness of its direction or relative interrelated structure. With the basic underlying conditioned reflex being that the author will arrive at the conclusion during the process of writing, it would follow that if one would finish the entire outline, the need to write the paper would become obsolete. This was not so much a rational thought as a deeply held emotional belief. The author, therefore, even with an outline, tended to go beyond and/or outside of it with subsequent and expectable difficulties to contain and limit herself to the outline of thought to which she committed herself initially.

The lack of conditioning in adhering to a firmly formulated outline 
compounds the difficulty in organizing sequential thought, whereas linear, logical progression of content is expected and examined by the Western reader. The process of writing a coherent paper is how the author attempted to present her arguments and persuade the reader using a contiguous line of reasoning. However, the author instead frequently experienced a randomly sequential or nonsequential outpouring of information or feelings as they would relate to reality or only to her state of mind. Therefore maintaining the analytical and objective perspective based on the predetermined structure of the outline was one of the most difficult parts of the process.

Already the outline itself was of ten formulated in an unorthodox manner as measured by Western standards. Initially, brief sentences were written down that were later to become important focal points of the subject under study. This process involved deep but of ten unfocused thought regarding relevant facts, principally unstructured contemplation and an accumulation of ideas which came into the writer's mind. After putting short sentences on paper or index cards, the author then proceeded to rearrange the pieces according to sequences logical to her in order that an organized outline would be crystallized.

But during the process of developing a coherent outline, the author tended to skip this process and supporting arguments to her assertions. The author either omitted by conditioned reflex answering "Why" questions or went on the assumption her reader would understand without the benefit of a full explanation. The questioning "Why" is the basis of scientific inquiry, but the author frequently had to overcome her tendency to answer a specific inquiry by simply stating that "it was that way", which would have resulted in a rambling and incoherent pattern 
and consequent gaps in the continuity of the investigation.

The author, during her time in America, has continuously experienced the basic difference in the attitude toward the degree of self-expression between United States and Japan. She of ten had to remind herself of the fact that writing the paper was a matter of genuine self-expression and that it was important to reveal her thoughts publicly regardless how trivial her ideas may have initially seemed to her. The author's shift of perspective, that one has an active choice to write what one believes to be correct, apart from that which one is simply expected to express, occurred during this period.

The constitution of the United States declares that every person has the right to free speech. As Aristotle states "...things that are true and things that are just have a natural tendency to prevail over their opposites" (McKeon, 1941. p.1327). People believe in the rationality of human beings and the natural force of truth and righteousness. In such a society people are expected to speak out for what they believe to be true and listen to what others believe to be right. Moreover, people are urged to be open-minded enough to respect and accept the opinions of others that have logical validity. Constant exposure to the exchange and the challenge of different opinions through discussion and debate result in the diversity of the people through a mutually educational process.

In a society where conformity is an essential value to maintain good human relationships, difference and originality of the individual is not encouraged. Instead, the government or the "authority" reserves the right to decide what it approves for people to believe in. As a result, an individual's apparent choices to determine what is desirable or not are few. In other words, the basic rationality of mankind is not realized, and 
therefore men who assume to know right and wrong within the logic suitable to their philosophy of government may actually take control over free speech for all practical purposes. Where individuals, when told what is right by a public authority, neither have much opportunity to be exposed to all sides of an issue nor the self-confidence to believe in that which they think to be right, then these people tend to give up on the responsibility to determine what is right or true, and there will be less individuality and self-direction than in a truly democratic society.

Japan adopted the concept of democracy after World War II, but the deep-rooted tradition from the past feudal system has not yet permitted her to become a true democratic nation. People in Japan are still seeking conformity, and have not generally learned to appreciate the benefits of free speech. Consequently, the Japanese educational system has not yet acknowledged the importance of debate and discussion, a useful means for people to become aware of their individual ability in seeking the truth through their belief in their own rationality. At present an argument, even for the sake of argument, might result in serious emotional conflicts among parties involved, such as a "loss of face", or in frustration from simply not knowing how to express thoughts sequentially. However, the author believes that the introduction and training of the theory of debate, argumentation and persuasion for the purpose of creating new skills in the sequential manner of presentation would help the Japanese communicate more effectively in an intercultural context.

The author's endeavor to use precise and clear language is worth mentioning. The clarity of the study depends largely upon the precision of language used by the author. It is therefore the author's responsibility to give clear boundaries to crucial terms. The systematic 
process of abstraction, which serves the process of defining, classifying, analyzing, and synthesizing, requires the same logical thoughts as an outline does.

But from her traditional point of view, the author was uncomfortable with extremely precise language. In Japan, the purpose of exchanging an opinion is not really to discuss the issue. A person simply wants to become acquainted with the slightly different view of others and let others know of his own, before adjusting his own opinion to be consistent with the rest of the group. Therefore, even in written discourse, the author was inclined to use successive general statements which could be interpreted in many ways.

All in all, the author experienced the process which allowed her to actualize her environmentally determined style of argument. It needs to be noted that an awareness of the dissimilarity is useful in shifting the perspective of the (group) self to one that has not been our own. Although it requires constant search for one's own communicative patterns and an effort to empathize, the process is certainly worth trying.

On the basis of the present study, the author thinks that the principle of the American approach to discourse (clarity) will serve as a reasonably good model for intercultural communication. Japanese need to recognize that communication is an essential means to solve and ameliorate currently existing problems. Since Japanese have been so immensely successful in adopting and improving foreign technology it should be possible and desirable to integrate the development of an elaborated communication competence into their effective technology.

Developing new communicative skills hand in hand with technological improvement and trade objectives will not only increase the Japanese 
impact in the American and world market, but it will result in greater self-confidence on the individual and group level. In the long run, increased options of communication style for all people will help bring about not only a greater degree of mutual acceptance but also an increased degree of co-equality that directly corresponds to the ability to effectively relate to all the other members in the family of nations. 


\section{BIBLIOGRAPHY}

Agee, Warren K., Phillip H. Ault and Edwin Emery. 1976. An Introduction to Mass Communication. 5th ed. New York: Harper \& Row Publishers.

Albert, Ethel M. 1964. "'Rhetoric,' 'Logic,' and 'Poetics' in Burundi: Culture Patterning of Speech Behavior." American Anthropologist. (The Ethnography of Communication: Special Publication.) 66. no.6. pt. 2. 35-54.

Asahi Shimbun. 1981. Feb. 1, Mar. 1, Apr. 1, May 1, Jun. 1, Jul. 1, Aug. 1, Sep. 1, Oct. 1, Nov. 1 .

Asante, Molefi K ., Eileen Newmark and Cecil A. Blake. eds. 1979. Handbook of Intercultural Communication. Beverly Hills : Sage Publication, Inc.

Barnlund, Dean C. 1975. Public and Private Self in Japan and the United States. Tok yo: The Simul Press.

Beniger, James R. and D. Eleanor Westney. 1981. "Japanese and U. S. Media: Graphics as a Reflection of Newspapers' Special Role." Journal of Communication. 31: no. 2. 14-27.

Bennett, Milton J. 1979. "Overcoming the Golden Rule: Sympathy and Empathy." Communication Yearbook. 3. ed. Dan Nimmo. International Communication Association.

Bernstein, Basil. 1964. "Elaborated and Restricted Codes: Their social Origins and Some Consequences." American Anthropologist. (The Ethnography of Communication: Special Publication.) 66: no.6 $5 \overline{5-69 .}$

Bogen, Joseph E. 1973. "The Other Side of the Brain: An Appositional Mind." in The Nature of Human Consciousness. ed. Robert E. Ornstein. 101-125.

Brembeck, Winston L. and W. S. Howell. 1952. Persuasion. A Means of Social Control. Englewood Cliffs : Prentice-Hall, Inc.

Casmir, Fred, and L.S. Harms. eds. 1970. International Studies of National Speech Education Systems. vol. 1. Minneapolis: Burgess Publishing Co.

Christian Science Monitor. 1981. Feb. 2, Mar. 3, Apr. 1, May 1, June 1, July 1, Aug. 1, Sep. 1, Oct. 1, Nov. 2.

Cleaver, Charles G. 1976. Japanese and Americans: Cultural Parallels and Paradoxes. Minneapolis: University of Minnesota Press. 
Cole, Michael, John Gay, Joseph A. Glick and Donald W. Sharp. 1970. The Cultural Context of Learning and Thinking---an Exploration in Experimental Anthropology. New York: Basic Books, Inc., Publishers.

Condon, John C., and Fathi S. Yousef. 1975. An Introduction to Intercultural Communication. In dianapolis: Bobb-Merrill.

- and Mitsuko Saito. eds. 1974. Intercultural Encounters with Japan. Tok yo: The Simul Press.

Deikman, Arthur J. 1973. "Bimodal Consciousness." in The Nature of Human Consciousness. ed. Robert E. Ornstein. 67-86.

Doi, Takeo. 1974. "Some Psychological Themes in Japanese Human Relationships." in Intercultural Encounters with Japan. eds. John C. Condon and Mits uko Saito. 17-26

Ewbank, Henry L., and J. Jeffery Auer. 1951. Discussion and Debate: Tools of a Democracy. 2nd ed. New York: Appleton-CenturyCrofts, Inc.

Gaggamiga, Michael S. 1973. "The Split Brain in Man." in The Nature of Human Consciousness. ed. Robert E. Ornstein. 87-100.

Glenn, Edmund S., D. Witmeyer and K.A. Stevenson. 1977. "Cultural Styles of Persuasion." International Journal of Intercultural Relations. 1. no. 3. 52-66.

Hall, Edward T. 1977. Beyond Culture. New York: Anchor Press.

Henderson, Harold G. 1958. An Introduction to Haiku. New York : Doubleday \& Company, Inc.

Holsti, Ole R. 1969. Content Analysis for the Social Sciences and Humanities. Massachuset ts: Addison Wesley Publishing Co.

Hsu, Francis L. K. ed. 1972. Psychological Anthropology. Massachuset ts : Schenkman Publishing Company, Inc.

Huber, Rober B. 1963. Influence through Argument. New York: David McKay Company, Inc.

Huruse, Noriko. 1978. "A Comparative Study of Communication Style in Japan and the United States as Revealed Through Content Analysis of Television Commercials." Unpublished Masters Thesis. Portland State University.

Inukai, Michiko. 1966. "Agreeing to Differ." Japan Quarterly. 13. no. $X$ 2. 182-183.

Isenhart, Myra W. 1980. "Intercultural Materials For an Argumentation Course." Communication Education. 29. no. 2 185-193. 
Johns, Ann McClelland. 1979. "A Comparison of Cohesive Elements in American Business and Non-Native Speaker Written Discourse." Unpublished Doctoral Dissertation. University of Southern California.

Kaplan, Robert B. 1966. "Cultural Thought Patterns in Intercultural Education." Language Learning. 16. nos. 1 \& 2. 1-20.

- 1971. The Anatomy of Rhetoric: Prolegomena to a Functional Theory of Rhetoric Essays for Teachers. Philadelphia: The Center for Curriculum Development, Inc.

- 1976. "A Further Note on Contrastive Rhetoric." Communication Quarterly. 24. no. 2. 12-19.

Katz, Bill, and Linda Sternberg Katz. 1982. Magazines for Libraries. New York: R.R. Bowker Co.

Kawashima, Takeyoshi. 1975. "The Status of the Individual in the Notion of Law, Right and Social Order in Japan." in The Japanese Mind. ed. Charles A. Moore. 262-287.

- 1979. "The Japanese Linguistic Consciousness and Law." Japan Echo. 6. no. 3. 105-114.

- and Wayne H. Oxford. 1970. "Speech Education in Japan." in International Studies of National Speech Education Systems. eds. Fred Casmir and L.R. Harms. 115-132.

Kinneavy, James L. 1980. A Theory of Discourse. New York: W. W. Norton Company.

Kobayashi, Toshihiro. 1976. "Toward True International Exchange." Japan Echo. 3. no. 4. 141-144.

Krippendorff, Klaus. 1980. Content Analysis: An Introduction to Its Methodology. Beverly Hills, California: Sage Publications, Inc.

Kunihiro, Masao. 1973. "Indigenous Barriers to Communication." The Japan Interpreter. 8. no. 1. 96-108.

- 1976. "The Japanese Language and Intercultural

Communication." The Japan Interpreter. 8. no. 1. 96-108.

Mainichi Shimbun. 1981. Oct. 1, Nov. 1.

Matsumoto, Michihiro. 1975. Chiteki Taiketsu no Ronri.

[Intellectual Persuasion.] Tokyo: Asahishuppan.

McBurney, James H., and Ernest J. Wrage, 1975. Guide to Good Speech. 4th ed. Englewood Cliff, New Jersey: Prentice-Hall, Inc.

McCroskey, James C. 1968. An Introduction to Rhetorical Communication. Englewood Cliff, New Jersey: Prentice Hall Inc. 
Mckeon, Richard P. 1941. The Basic Works of Aristotle. New York: Random House.

Mehrabian, Albert. 1971. Silent Messages. Belmont, California: wadsworth Publishing Co., Inc.

Miller, Gerald R, and Henry E. Nicholson. 1976. Communication Inquiry: A Perspective on a Process. Massachuset ts: Addison-Wesley Publishing Co.

Moore, Charles. ed., 1975. The Japanese Mind: Essentials of Japanese Philosophy and Culture. An East-West Center Book. Honolulu: The University Press of Hawail.

Moran, Louis J. 1973. "Comparative Growth of Japanese and North American Cognitive Dictionaries." Child Development. 44. no. 4. 862-865.

Mushakoji, Kinhide. 1976. "The Cultural Premises of Japanese Diplomacy." in The Silent Power. ed. Japan Center for International Exchange. 35-49.

Nadeau, Ray E. 1972. Modern Rhetoric of Speech Communication. 2nd ed. Massachuset ts : Addison-Wesley Publishing Company, Inc.

Nakamura, Hajime. 1974. The Ways of Thinking of Eastern Peoples. India, China, Tibet, Japan. Honolulu: The University Press of Hawaii.

Nakane, Chie. 1972. Tekio no Joken. [The Propensity for Adaptation] Tokyo: Kodansha.

New York Times. 1981. Feb. 1, Mar. 1, Apr. 1, May 1, June 1, July 1, Aug. 1, Sep. 1, Oct. 1, Nov. 1.

Nishio, Minoru. 1957. Nihonjin no Kotoba. [Language of the Japanese.] Tok yo: Iwanami Shoten.

Norbeck, Edward and George DeVos. 1972. "Culture and Personality: The Japanese." in Psychological Anthropology. ed. Francis L.K. Hsu.

Oide, Akira. 1965. Nihongo to Ronri. [Japanese Language and its Logic.] Tok yo: Kodansha.

Okabe, Roichi. 1981. "Cultural Assumption of Communication Theory From Eastern and Western Perspective, the Case of Japan and the United States" Paper presented at Speech Communication Association. Anaheim, California. November.

Oliver, Robert T. 1971. Communication and Culture in Ancient India and China. New York: Syracuse University Press. - 1974. "Asian Public Address and Comparative Public Address." The Speech Teacher. 23. no. 2. 101-108. 
Ornstein, Robert E. ed. 1973. The Nature of Human Consciousness. San Francisco: W.H. Freeman and Company.

Pribram, Karl H. 1949. Conflicting Patterns of Thought. Washington: Public Affairs Press.

Rosovsky, Henry. ed. 1972. Discord in the Pacific. Challenges to the Japanese-American Alliance. The American Assembly, Columbia University.

Ruesch, Jurgen and Gregory Bateson. 1968. Communication: The Social Matrix of Psychiatry. New York: W. W. Norton Co. Inc.

Samovar, Larry A. and Richard E. Porter. eds. 1976. 1982.

Intercultural Communication: A Reader. Belmont, California: Wadsworth Publishing Co., Inc.

Siegel, Sidney. 1956. Nonparametric Statistics for the Behavioral Sciences. New York: McGraw-Hill Book Co., Inc.

Stewart, Edward C. 1974. Dimention of International Education. No.3 American Cutural Patterns: A Cross-Cultural Perspective. Pittsburg: Regional Council for International Education.

Strunk, William Jr. and E. B. White. 1979. The Elements of Style. 3rd ed. New York: MacMillan Co..

Suzuki, Takao. 1975. Tozasareta Gengo; Nihongo no Sekai. [Japanese: A Language in Isolation.] Tok yo: Shinchosha.

Tanizaki, Junichiro. 1975. Bunsho Tokuhon. [A Literary Composition Reader.] Tokyo: Chuokoron.

Toulmin, Stephen E., Richard Rieke and Allan Janik. 1979. An Introduction to Reasoning. New York: MacMillan Publishing Co., Inc.

Toyama, Shigehiko. 1976. Nihongo no Kosei. [The Characteristics of the Japanese Language. Tokyo: Kodansha.

Van Zandt, Howard F. 1976. "How to Negotiate in Japan." in Intercultural Communication: A Reader. 2nd ed. edited by Larry A. Samovar and Richard E. Porter. 307-318

Washington Post. 1981. Aug. 1, Sep. 1.

Watzlawick, Paul, Janet H. Beavin, and Donald D. Jackson. 1962. Pragmatics of Human Communication. New York: W. W. Norton Co. Inc.

Williams, Redrick. 1968. Reasoning with Statistics: Simplified Examples in Communication Research. New York: Holt, Rinehart and Winston Inc. 
Windes, Russel R. and Arthur Hastings. 1965. Argumentation and Advocacy. New York: Random House.

Yamamoto, Akira and Akira Fujitake. 1980. Nihon no Masucominikeishon

[Mass Communication in Japan.] Tok yo: Nihon Hoso Shuppan.

Yomiuri Shimbun. 1981. Feb. 1, Mar. 1, Apr. 1, May 1, June 1, July 1, Aug. 1, Sep. 1, Oct. 1, Nov. 1 . 


\section{APPENDIX A}

\section{INSTRUCTIONS FOR RATERS}

The following articles A to $\mathrm{F}$ are randomly chosen from newspapers. After reading each article, please rate each argument according to the degree to which they fall into the following categories. A scoring sheet is attached.

First category : linear - nonlinear

Each article should be analyzed in terms of whether or not the presentation of the argument is linear. A linear presentation is here defined as a succession of steps that are dependent upon the previous supposition. A nonlinear presentation consists of statements that are neither related internally nor dependent upon the progression of statements.

Second category: inductive reasoning - deductive reasoning

Each article should be analyzed in terms of whether it is inductive reasoning or deductive reasoning. Inductive reasoning is defined as reasoning which proceeds from the particular case or cases to the general rule. Inductive reasoning, therefore is to establish the general rule. Induction is the process of observation of facts and verification of the 
observation. Deductive reasoning is defined as reasoning which proceeds from the general rule to the particular case. Deduction is the process to check the implication of the hypothesis or general rule with the particular case or fact.

Third category: explicit - implicit

Each article should be analyzed as to whether conclusions are explicitly stated or implied. Conclusion is any belief that a writer wishes $h$ is reader to accept.

Fourth category: objective - subjective

The evidence used in each article should be studied as to whether it is subjective or objective. Criteria for subjective evidence include personal opinions, common or generally accepted knowledge, and personal feelings toward the subject matter. Criteria for objective evidence include statistical data, detailed accumulation of facts, experimental observations, previously established conclusions and personal expert testimony with a justifying underlying basis.

Fifth category: analytical - emotional

Each article should be analyzed as to whether it includes more analytical statements than it does emotional statements. Analytical statements are defined as logical statements which separate or break up the subject into its constituent and express the author's rational thoughts 
rather than his emotion. Emotional statements are defined as expression of the author's personal feelings which do not necessarily accompany logic.

Sixth category: concrete - abstract

Each article should be examined as to whether statements are concrete or abstract. Concrete statements are characterized by the use of the writer's immediate observable experiences, therefore are mainly descriptive, specific and particular. Abstract statements are disassociated from any specific instance and personal input and deal with subject more theoretically.

Seventh category: direct - indirect

Each article should be analyzed whether the writer of the article comes to the point immediately or not. The relative direct introduction of the subject is rated if the author discusses the subject matter from the first paragraph ( if the topic is about a matter preciously discussed, the explanation about the discussed topic is not counted). The relative indirect introduction of the subject is rated if the author uses the introductory paragraphs that do not necessarily concern the immediate subject matter but of fer background arguments or lead-ins. 
Eighth category: no conclusion - once - more than once

Each article should be analyzed as to whether the conclusion is stated once, more than once, or not at all.

Nineth category: wide perspective - narrow perspective

Each article should be analyzed as to whether the author uses a wide perspective toward the subject matter or not. A wide perspective is defined as giving a multiple evidence base to draw a conclusion. A narrow perspective is giving a singular or small evidence base toward the subject's conclusion.

Tenth category: definite - conditional

Each article should be analyzed as to whether statements accompany more definite and strengthening qualifiers than they do conditional and weakening qualifiers. Definite qualifiers include absolutely, definitely, certainly, necessarily etc. Conditional qualifiers include presumably, probably, maybe, very likely, plausibly, seemingly, possibly etc. 
SCORING SHEET

Article ( )

\#1 Linear

Nonlinear

\begin{tabular}{lllllll}
\hline-3 & -2 & -1 & 0 & 1 & 2 & 3
\end{tabular}

\#2 Inductive

Deductive

\begin{tabular}{lllllll}
\hline-3 & -2 & -1 & 0 & 1 & 2 & 3
\end{tabular}

\#3 Explicit

Implicit

$\begin{array}{lllllll}-3 & -2 & -1 & 0 & 1 & 2 & 3\end{array}$

\#4 Objective

Subjective

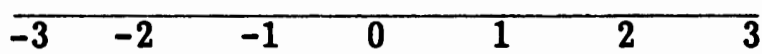

\#5 Analytical

Emotional

\begin{tabular}{lllllll}
\hline-3 & -2 & -1 & 0 & 1 & 2 & 3
\end{tabular}

\#6 Concrete

Abstract

\begin{tabular}{llllllll}
\hline-3 & -2 & -1 & 0 & 1 & 2 & 3
\end{tabular}

\#7 Direct

Indirect

\begin{tabular}{lllllll}
\hline-3 & -2 & -1 & 0 & 1 & 2 & 3
\end{tabular}

\#8 No conclusion Once Repeated

\begin{tabular}{lllllll}
\hline-3 & -2 & -1 & 0 & 1 & 2 & 3
\end{tabular}

\#9 Wide perspective Narrow perspective

\begin{tabular}{llllllll}
\hline-3 & -2 & -1 & 0 & 1 & 2 & 3
\end{tabular}

\#10 Definite Conditional

\begin{tabular}{lllllll}
\hline-3 & -2 & -1 & 0 & 1 & 2 & 3
\end{tabular}




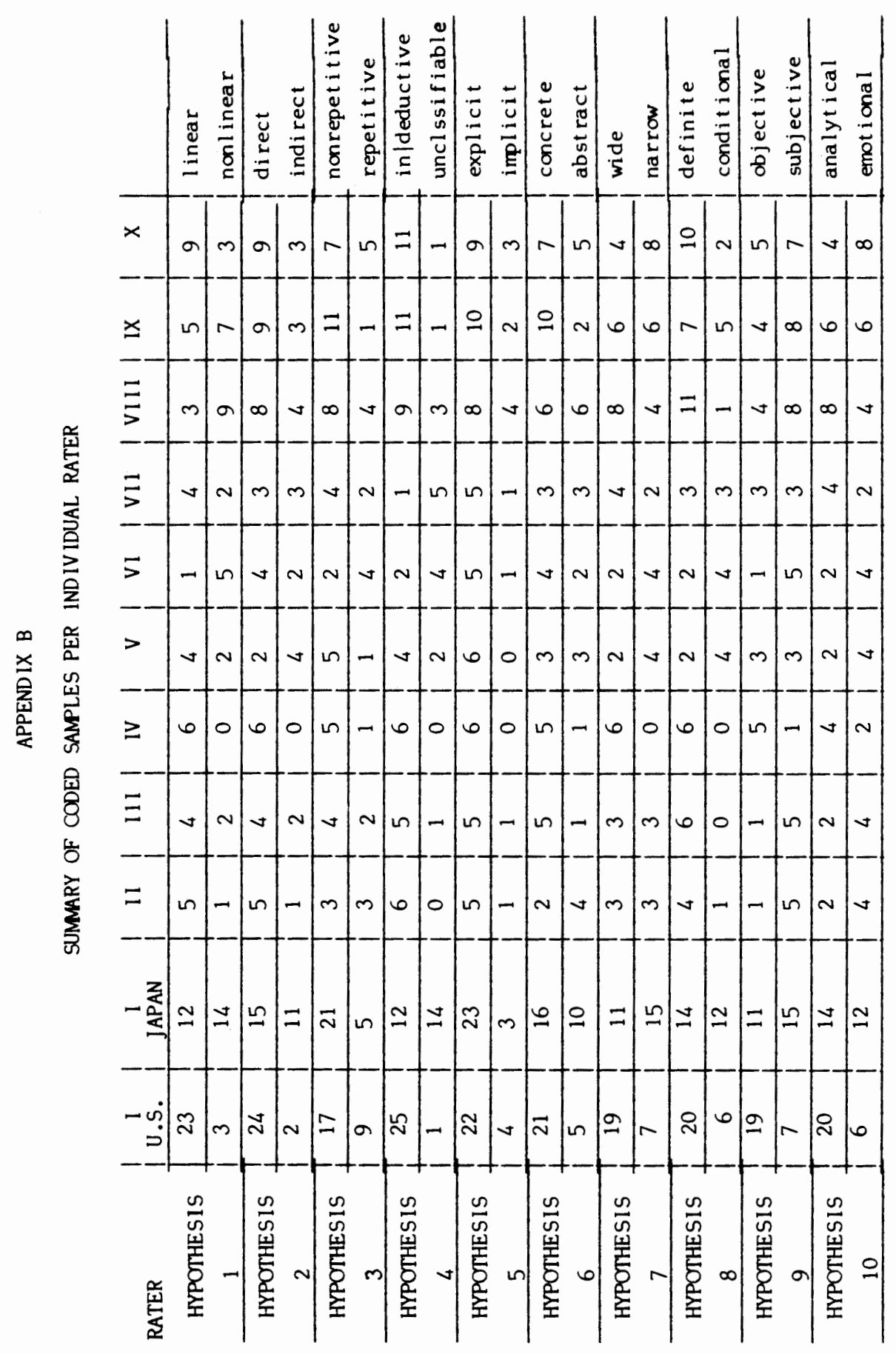

